This document is confidential and is proprietary to the American Chemical Society and its authors. Do not copy or disclose without written permission. If you have received this item in error, notify the sender and delete all copies.

\title{
Complex self-assembly behavior of bis-hydrophilic PEO-b- PCL-b-PMOXA triblock copolymers in aqueous solution
}

\begin{tabular}{|r|l|}
\hline Journal: & Macromolecules \\
\hline Manuscript ID & ma-2017-01498h.R1 \\
\hline Manuscript Type: & Article \\
\hline Domplete List of Authors: & $\begin{array}{l}\text { Konishcheva, Evgeniia; University of Basel, Chemistry } \\
\text { Zhumaev, Ulmas; Max-Planck-Institut fur Polymerforschung } \\
\text { Kratt, Maximilian; University of Basel } \\
\text { Oehri, Valentin; University of Basel } \\
\text { Meier, Wolfgang; University of Basel, Department of Chemistry }\end{array}$ \\
\hline
\end{tabular}

\section{SCHOLARONE \\ Manuscripts}




\title{
Complex self-assembly behavior of bis-hydrophilic PEO-b-PCL-b-PMOXA triblock copolymers in aqueous solution
}

Evgeniia V. Konishcheva ${ }^{\dagger^{*}}$, Ulmas E. Zhumaev ${ }^{\ddagger}$, Maximilian Kratt ${ }^{\dagger}$, Valentin Oehri ${ }^{\dagger}$, Wolfgang Meier ${ }^{* *}$

†Department of Chemistry, University of Basel, Klingelbergstrasse 80, 4056 Basel, Switzerland

${ }^{\ddagger}$ Molecular Spectroscopy Department, Max Planck Institute for Polymer Research, Ackermannweg 10, 55128 Mainz, Germany

*Corresponding authors: ev.konishcheva@gmail.com,wolfgang.meier@unibas.ch

\begin{abstract}
We report aqueous self-assembly of linear amphiphilic triblock copolymers poly(ethylene oxide)-block-polycaprolactone-block-poly(2-methyl-2-oxazoline) (PEO-bPCL- $b$-PMOXA) and their PEO- $b$-PCL precursors with different PCL and PMOXA block lengths using three preparation methods: film rehydration, solvent evaporation, and cosolvent. For PEO- $b$-PCL, the self-assembled structures were the ordinary spherical particles and polymersomes. For PEO- $b$-PCL- $b$-PMOXA, we observed polymersomes with asymmetric membrane, cloud-like aggregates, elongated particles, Y-shaped elongated particles, and 3D networks. All structures were of micrometer size and characterized using laser scanning microscopy (LSM). 3D networks were also characterized using z-stack confocal LSM, transmission electron microscopy (TEM), and cryogenic TEM. We demonstrated that film rehydration method results in pseudoequilibrium structures, whereas structures formed using solvent evaporation and co-solvent methods are under kinetic control. We showed how kinetically controlled structures can be transformed into pseudoequilibrium morphologies.
\end{abstract}




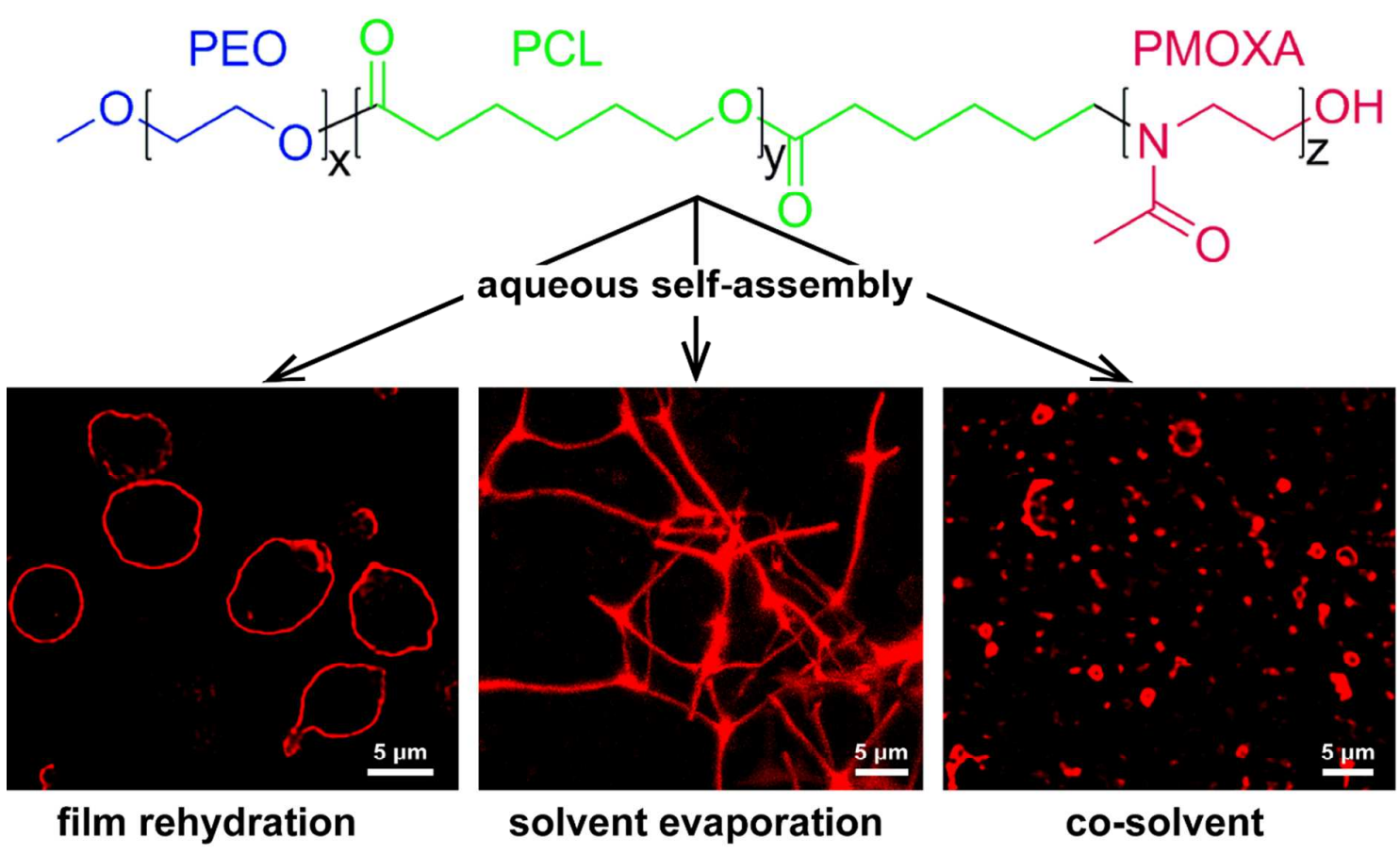




\section{Introduction}

Complex structural organization achieved through noncovalent interactions leads to unique functions (e.g. DNA, RNA) and very specific catalytic activity (e.g. enzymes) of natural macromolecules. Mimicking such complex structural organization by self-assembly of synthetic molecules is a promising approach towards the rational design of programmable functional (nano)materials. Water is a unique solvent ${ }^{1}$ for self-assembly based on noncovalent interactions, and structures self-assembled in aqueous solution are of particular importance for biomedical applications, such as drug delivery and tissue engineering. Low molecular weight surfactants and amphiphilic block copolymers are prominent example of synthetic molecules that self-assemble into various structures in water. Self-assembly of these synthetic molecules is driven by hydrophobic interactions. Amphiphilic block copolymers are advantageous over low molecular weight surfactants and natural lipids due to the higher stability of the self-assembled structures ${ }^{2}$ and possibility to tune properties of the aggregates by changing composition and length of the blocks. Such a tuning procedure is becoming more and more straightforward thanks to the fast development of polymerization techniques and vast availability of different monomers. ${ }^{3-5}$

Linear $\mathrm{AB}$ diblock copolymers, where $\mathrm{A}$ and $\mathrm{B}$ stand for the hydrophilic and hydrophobic blocks, respectively, are the most explored type of amphiphilic block copolymers. It has been found that aqueous self-assembled structures of linear AB polymers can be described in terms of the packing geometry of an individual polymer molecule, and in most cases such structures formed in dilute solutions (few wt \%) are limited to micelles, rods, and polymersomes. ${ }^{6-13}$ Introduction of the third, hydrophilic C block, can break this limitation and facilitate the access of more complex self-assembled structures that are not accessible with conventional linear $\mathrm{AB}$ polymers. However, there is lack of experimental data on aqueous self-assembly of linear ABC triblock copolymers. Consequently, in contrast to linear 
$\mathrm{AB}$ polymers, in the case of linear $\mathrm{ABC}$ polymers it is not known how hydrophilic weight fraction and conditions of self-assembly influence morphology of the self-assembled structures.

Only few works report about aqueous self-assembly of linear ABC polymers. Some of them do not represent more complex self-assembly behavior of $\mathrm{ABC}$ compared to $\mathrm{AB}$ polymers, since the observed structures are ordinary micelles, rods, and polymersomes. ${ }^{14-15}$ The other works do represent more complex self-assembly behavior by reporting polymersomes with asymmetric membrane ${ }^{16-24}$ and structures with domains in the corona and stimuli-responsive blocks. ${ }^{25-28}$ Aqueous self-assembled structures formed by linear ABC polymers can be more diverse, as suggested by the recent studies devoted to the selfassembly of linear triblock copolymers in organic solvents. ${ }^{29-30}$ The structures possessed various morphologies with domains in the core, e.g. spheres on spheres, spheres on rods, rods on vesicles, spheres on vesicles, etc. However, these structures were observed for linear ABC polymers in organic $\mathrm{C}$-selective solvent, and the established rules are not necessarily applicable for (A and C)-selective solvent, i.e. for water in the case of hydrophilic A and C blocks.

In this work, we present the first systematic investigation of self-assembly of linear bis-(A, C)-hydrophilic ABC polymers in dilute aqueous solution. Our aim is to gain insight into the effect of hydrophilic weight fraction and conditions of self-assembly on the morphology of the self-assembled structures. As a first step towards the major aim, we explore the latter effects in dilute aqueous solution (0.2 wt \%). As a model ABC system, we choose recently developed by us narrowly dispersed $\left(\mathrm{Ð}_{\mathrm{M}}<1.25\right)$ poly(ethylene oxide)-blockpolycaprolactone-block-poly(2-methyl-2-oxazoline) (PEO- $b$-PCL- $b$-PMOXA) polymers. ${ }^{16}$ Since the synthesis of PEO- $b$-PCL- $b$-PMOXA is free of toxic reagents, PEO and PMOXA are biocompatible hydrophilic blocks, and PCL is biodegradable hydrophobic block, the 
reported self-assembled structures might serve as potential candidates for biomedical applications. We tune the hydrophilic weight fraction of PEO- $b$-PCL- $b$-PMOXA by changing PCL and PMOXA block lengths, while keeping PEO block length constant (45 units), and explore structures resulting from three self-assembly methods/conditions, namely film rehydration, solvent evaporation, and co-solvent. To explain the formation of the observed structures assembled from PEO- $b$-PCL- $b$-PMOXA, we compare them with self-assembled structures formed by their precursors, PEO- $b$-PCL, under the same self-assembly conditions. Finally, to understand whether the observed structures are under thermodynamic or kinetic control, we investigate how change of details in self-assembly procedures, such as temperature, rate of the co-solvent addition, stirring rate, and self-assembly duration, affects the morphology of the self-assembled structures. 


\section{Experimental part}

Experimental details on methods (NMR, GPC, DSC, TEM, cryoTEM) and synthesis of PEO- $b$-PCL ${ }^{31}$ and PEO- $b$-PCL- $b-$ PMOXA $^{16}$ can be found in related publications and Supporting Information.

Laser scanning microscopy (LSM). LSM images were recorded on an inverted Zeiss LSM510 META/ConfoCor 2 FCS microscope using a 100× 1.4 Oil Plan-Apochromat DIC objective lens. Bodipy 630/650 and calcein disodium salt were excited by the $633 \mathrm{~nm} \mathrm{He}-\mathrm{Ne}$ laser line (10\% output) and $488 \mathrm{~nm}$ Argon laser line (10\% output), respectively. The excitation light was passed through a HFT UV 488/543/633 beam splitter. The emission light from Bodipy 630/650 was passed through a LP 650, and the signal from calcein through a BP 474-525. The transmittance signal was recorded simultaneously on a T-PMT detector. 12 bit images of $1024 \times 1024$ pixels were recorded at a scan speed of $51.20 \mu$ s per pixel. z-Stack confocal LSM (CLSM) was performed on an inverted Zeiss LSM880 using a $63 \times 1.4$ Oil Plan-Apochromat DIC M27 objective. Bodipy 630/650 was excited by the $633 \mathrm{~nm} \mathrm{He}-\mathrm{Ne}$ laser line. To get z-stacks with identical signal intensity, the laser power was increased from $0.1 \%$ to $3 \%$ output over the z-scan range. The excitation light was passed through a HFT UV 488/543/633 beam splitter. The emission light was passed through a LP 650 and recorded on PMT detector. The pinhole was set to 1 airy unit, and the z-slices were recorded with $0.5 \mu \mathrm{m}$ step enabling $50 \%$ overlap for efficient $3 \mathrm{D}$ reconstruction. 16 bit images of $1280 \times 1280$ pixels were recorded at a scan speed of $13.11 \mu$ s per pixel. $5 \mu 1$ of a stained sample were placed onto a $22 \mathrm{~mm} \times 50 \mathrm{~mm}$ glass cover slip, covered with a round $(\varnothing 13 \mathrm{~mm})$ cover slip, and sealed with nail polish. The LSM images were processed with ImageJ and its LSM toolbox plug-in. The average diameter of polymersomes was calculated from area $(\mathrm{d}=$ $\left.2(\mathrm{~s} / \pi)^{1 / 2}\right)$ determined for each individual particle using "Analyze Particles" option based on at least 3 different images (at least 300 objects in total). Length of elongated structures was 
determined as Feret's diameter based on at least 3 different images (at least 70 objects in total). z-Stack CLSM images were recorded in ZEN software, deconvoluted in Huygens software, and z-projection was performed in ImageJ software using Z Projection plug-in.

Self-assembly. Three methods of self-assembly were employed to obtain microscale structures of PEO- $b$-PCL and PEO- $b$-PCL- $b$-PMOXA: film rehydration, solvent evaporation, and co-solvent. In all experiments we used $2 \times 5 \mathrm{~mm}$ PTFE magnetic stir bars, and final polymer concentration was $0.2 \mathrm{wt} \%$. Microscale structures $(50 \mu \mathrm{l})$ were stained with $0.5 \mu \mathrm{l}$ of $0.72 \mu \mathrm{M}$ Bodipy $630 / 650$ prior to visualization by LSM. Possible effect of Bodipy $630 / 650$ on self-assembled structures was tested by comparing bright field images of the assemblies formed by $\mathrm{E}_{45} \mathrm{C}_{110} \mathrm{M}_{4}$ and $\mathrm{E}_{45} \mathrm{C}_{153} \mathrm{M}_{11}$ before and after dye addition. No visible differences in self-assembled structures were observed, suggesting no effect of fluorescent dye on the morphology of the self-assembled structures, at least on the experimental time scale (data not shown).

Film rehydration. $2 \mathrm{mg}$ of a polymer was dissolved in $200 \mu \mathrm{l}$ of $\mathrm{CH}_{2} \mathrm{Cl}_{2}$ and transferred into a $5 \mathrm{ml}$ glass round-bottom flask. $\mathrm{CH}_{2} \mathrm{Cl}_{2}$ was slowly removed by rotary evaporation $\left(110 \mathrm{rpm}, 40^{\circ} \mathrm{C}, 710 \mathrm{mbar}\right)$, the polymer film was dried for $30 \mathrm{~min}(110 \mathrm{rpm}$, $40{ }^{\circ} \mathrm{C}, 5 \mathrm{mbar}$ ), and $1 \mathrm{ml}$ of Milli-Q water was added for the rehydration. The samples were placed into an oil bath which was quickly heated by a heating plate to $62{ }^{\circ} \mathrm{C}\left(3{ }^{\circ} \mathrm{C} \cdot \mathrm{min}^{-1}\right)$. The samples were stirred at $500 \mathrm{rpm}$ for $24 \mathrm{~h}$ at $62{ }^{\circ} \mathrm{C}$. After $24 \mathrm{~h}$ the oil bath $\left(62{ }^{\circ} \mathrm{C}\right)$ was removed, leading to a rapid decrease of the sample temperature to $22{ }^{\circ} \mathrm{C}$. In the experiments where the heating rate was controlled, the temperature was increased by steps of $10^{\circ} \mathrm{C}$ $\left(1.5^{\circ} \mathrm{C} \cdot \mathrm{min}^{-1}\right)$ from $22^{\circ} \mathrm{C}$ to $62{ }^{\circ} \mathrm{C}$, and the samples were left equilibrating at each temperature for $1 \mathrm{~h}$. After incubation for $24 \mathrm{~h}\left(500 \mathrm{rpm}, 62^{\circ} \mathrm{C}\right)$, the solutions were cooled to $22{ }^{\circ} \mathrm{C}$ by removing the oil bath. The experiments where the cooling rate was controlled, the samples were placed into an oil bath which was quickly heated by a heating plate to $62{ }^{\circ} \mathrm{C}$ 
$\left(3{ }^{\circ} \mathrm{C} \cdot \mathrm{min}^{-1}\right)$, incubated for $24 \mathrm{~h}\left(500 \mathrm{rpm}, 62^{\circ} \mathrm{C}\right)$, and the temperature was decreased by steps of $10{ }^{\circ} \mathrm{C}\left(0.6{ }^{\circ} \mathrm{C} \cdot \mathrm{min}^{-1}\right)$ from $62{ }^{\circ} \mathrm{C}$ to $22^{\circ} \mathrm{C}$, and the samples were left equilibrating at each temperature for $1 \mathrm{~h}$.

Solvent evaporation. $2 \mathrm{mg}$ of a polymer was dissolved in $200 \mu \mathrm{l}$ of $\mathrm{CH}_{2} \mathrm{Cl}_{2}$ and transferred into a $2.5 \mathrm{ml}$ glass vial, and then $1 \mathrm{ml}$ of Milli-Q water was added in one shot. The mixtures were left open for the evaporation of $\mathrm{CH}_{2} \mathrm{Cl}_{2}\left(350 \mathrm{rpm}, 24 \mathrm{~h}, 22{ }^{\circ} \mathrm{C}\right)$ and covered with a $500 \mathrm{ml}$ beaker to avoid contamination of the samples. To check whether the structures formed in solvent evaporation method can undergo a morphological transition to the ones formed in film rehydration method, different structures formed by polymers with different PCL and PMOXA lengths, i.e. $\mathrm{E}_{45} \mathrm{C}_{110} \mathrm{M}_{4}, \mathrm{E}_{45} \mathrm{C}_{135} \mathrm{M}_{4}$, and $\mathrm{E}_{45} \mathrm{C}_{135} \mathrm{M}_{20}$, were incubated at $62{ }^{\circ} \mathrm{C}$ for $24 \mathrm{~h}$ at $350 \mathrm{rpm}$ after evaporation of $\mathrm{CH}_{2} \mathrm{Cl}_{2}$.

Co-solvent. $2 \mathrm{mg}$ of a polymer was dissolved in $200 \mu \mathrm{l}$ of THF and added dropwise $\left(\sim 200 \mu \mathrm{l} \cdot \mathrm{min}^{-1}\right)$ into $800 \mu \mathrm{l}$ of Milli-Q water stirring at $350 \mathrm{rpm}$ in a $2.5 \mathrm{ml}$ glass vial. The vials were closed and the mixture was left stirring at $350 \mathrm{rpm}$ for $24 \mathrm{~h}$ at $22{ }^{\circ} \mathrm{C}$. To check the influence of the co-solvent nature on self-assembly, self-assembly of $\mathrm{E}_{45} \mathrm{C}_{153} \mathrm{M}_{4}$ was performed using DMF / ACN / acetone as a co-solvent, since these solvents are among those able to solubilize PEO- $b$-PCL- $b$-PMOXA copolymers. For the encapsulation experiments, $10 \mathrm{mM}$ solution of calcein disodium salt was used instead of water. After the self-assembly the solution was placed into a dialysis membrane (RC, MWCO 3.5-5 kDa, SpectraPor) and dialyzed against 1 L of Milli-Q water for 3 days (solution was exchanged 9 times). 


\section{Results and Discussion}

Bis-hydrophilic PEO- $b$-PCL- $b$-PMOXA triblock copolymers (Scheme 1) with fixed PEO length (45 units / $2 \mathrm{kDa})$ and various PCL (48 - 153 units / $5.5-17.4 \mathrm{kDa})$ and PMOXA ( $3-25$ units / $0.2-2.1 \mathrm{kDa})$ lengths were tested for the ability to self-assemble in aqueous solution using film rehydration, solvent evaporation, and co-solvent methods. In all self-assembly methods final polymer concentration was $0.2 \mathrm{wt} \%$. All polymers selfassembled into microscale structures which were characterized by LSM. We discuss the observed structures in the following subsections devoted to each self-assembly method. Further in the text, PEO- $b$-PCL- $b$-PMOXA polymers are abbreviated as $\mathrm{E}_{\mathrm{x}} \mathrm{C}_{\mathrm{y}} \mathrm{M}_{\mathrm{z}}$, where $\mathrm{x}, \mathrm{y}$, and $\mathrm{z}$ denote the number of monomer units of PEO, PCL, and PMOXA, respectively.

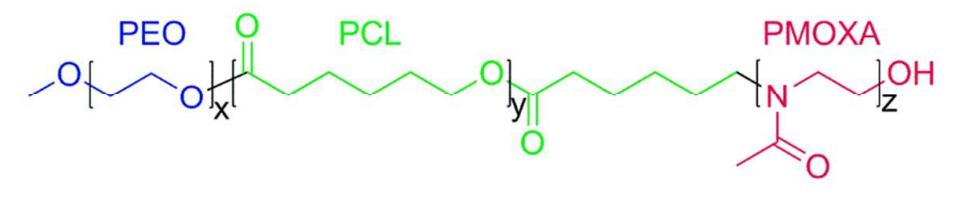

Scheme 1. Structure of PEO- $b$-PCL- $b$-PMOXA polymers.

Film rehydration method. In film rehydration method, a polymer was dissolved in $\mathrm{CH}_{2} \mathrm{Cl}_{2}$ and placed in a round-bottom glass flask. A thin polymer film was formed on the wall of the glass flask by removing $\mathrm{CH}_{2} \mathrm{Cl}_{2}$ by rotary evaporation. The film was rehydrated for $24 \mathrm{~h}$ after addition of water. Rehydration was performed at $62{ }^{\circ} \mathrm{C}$ due to the semicrystalline nature of the PCL block. The melting temperature of PEO- $b$-PCL- $b$-PMOXA polymers is $\mathrm{T}_{\mathrm{m}} \approx 61{ }^{\circ} \mathrm{C}$ (DSC Fig. S1). Self-assembly did not occur at $55^{\circ} \mathrm{C}$, and the polymers remained as a precipitate.

Fig. 1A depicts the predominant structures formed by PEO- $b$-PCL- $b$-PMOXA and their precursors, PEO- $b$-PCL, where each structure corresponds to the polymer with a certain molecular weight $\mathrm{M}_{\mathrm{n}}$ (or length) of PCL and hydrophilic weight fraction $f$ :

$$
f=\frac{M_{\text {hydrophilic }}}{M_{\text {hydrophilic }}+M_{\text {hydrophobic }}}=\frac{M_{n}(P E O)+M_{n}(P M O X A)}{M_{n}(P E O)+M_{n}(P M O X A)+M_{n}(P C L)}
$$


Most of PEO- $b$-PCL- $b$-PMOXA polymers formed two or three types of structures (e.g. Fig. S2), but for simplicity reasons, only predominant structures are plotted in Fig. 1A. More detailed information on morphology of the self-assembled structures can be found in Table S1. Here, in Fig. 1A, and in the following morphology diagrams, points of the same color represent polymers with similar PMOXA lengths. For simplicity, we combined close values of PMOXA lengths in groups indicated by dashed lines, e.g. the lengths of 7-12 units belong to the group PMOXA 10. Such combination is reasonable taking into account the dispersity of our triblock copolymers $\left(1.10<\mathrm{Ð}_{\mathrm{M}}<1.25\right.$, Table S1). Point shapes in morphology diagrams indicate certain morphologies. The gray areas point out regions of the same morphology.
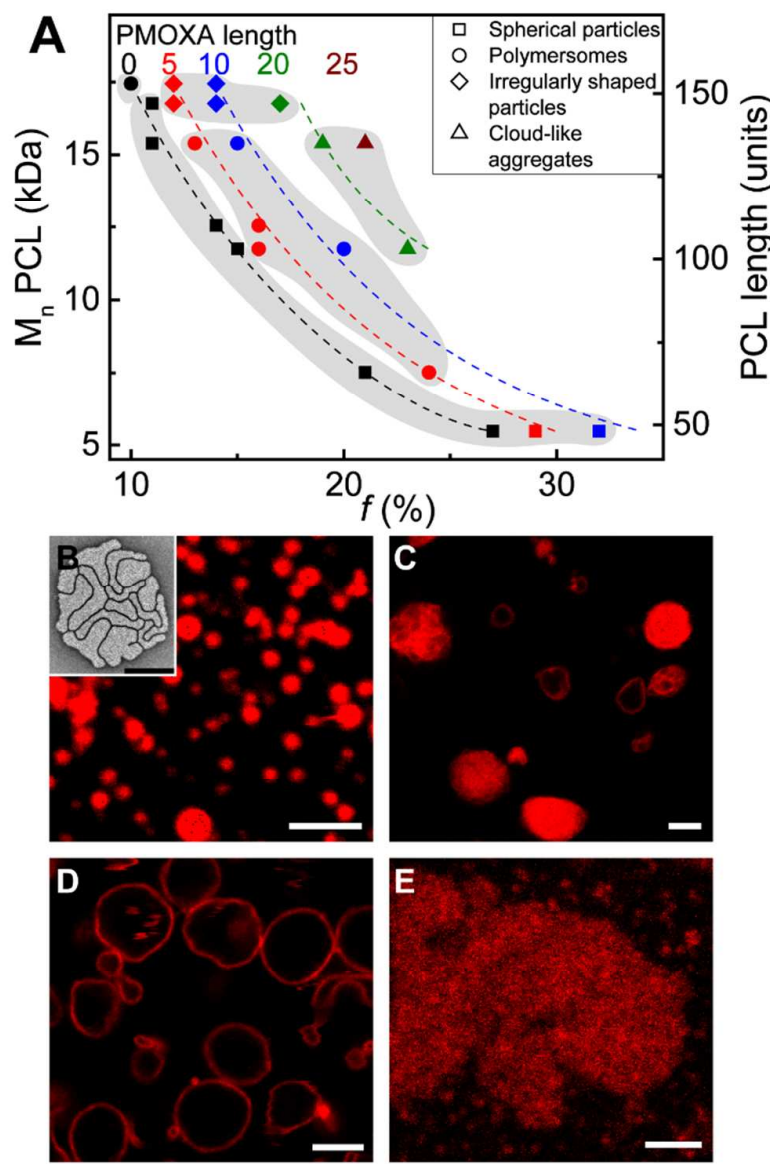

Figure 1. Self-assembled structures observed in film rehydration method. (A) Morphology diagram showing structures formed by PEO- $b$-PCL and PEO- $b$-PCL- $b$-PMOXA in aqueous 
solution as a function of the molecular composition. Points of each color correspond to polymers with a certain PMOXA length. Points of each shape correspond to a certain morphology: spherical particles (squares), polymersomes (circles), irregularly shaped particles (diamonds), cloud-like aggregates (triangles). The gray areas point out regions of the same morphology. Representative LSM images of the structures formed by (B) $\mathrm{E}_{45} \mathrm{C}_{147}-$ spherical particles, (C) $\mathrm{E}_{45} \mathrm{C}_{147} \mathrm{M}_{18}$ - irregularly shaped particles, (D) $\mathrm{E}_{45} \mathrm{C}_{110} \mathrm{M}_{4}$ polymersomes, (E) $\mathrm{E}_{45} \mathrm{C}_{135} \mathrm{M}_{20}$ - cloud-like aggregates. Structures were stained with Bodipy $630 / 650$. Scale bars are $5 \mu \mathrm{m}$. B inset is a representative TEM image of negatively stained spherical particles formed by $\mathrm{E}_{45} \mathrm{C}_{147}$; scale bar is $200 \mathrm{~nm}$.

In the absence of PMOXA block, i.e. in the case of PEO- $b$-PCL, morphology of the self-assembled structures changes from polymersomes to spherical particles (Fig. 1B) with an increase in $f$. These spherical particles with diameters 0.2-2 $\mu \mathrm{m}$ (determined from TEM) are composed of distinct domains, and the domain have shapes ranging from spheres to rods (Fig. 1B inset, S3). Perhaps such domains aggregate into spherical particles while cooling of the solution and crystallization of PCL block, but in this case one would expect formation of particles with rather irregular shape. In the case of PEO- $b$-PCL- $b$-PMOXA with PMOXA length of 5 and 10 units, morphology of the structures changes in the row irregularly shaped particles (Fig. 1C) - polymersomes (Fig. 1D) - spherical particles with an increase in $f$. In the case of PEO- $b$-PCL- $b$-PMOXA with PMOXA length of 20 units, morphology of the selfassembled structures changes from irregularly shaped particles to cloud-like aggregates (Fig. 1E) with an increase in $f$. Revealing trends between morphology of self-assembled structures and $f$ is a common practice in studies of self-assembly of diblock copolymers. For PEO- $b$ PCL in film rehydration method, the trend is known to be irregularly shaped particles polymersomes - spherical particles with increasing $f .^{32}$ Our observed trend for PEO- $b$-PCL follows this literature trend for PEO-b-PCL diblock copolymers (Fig. 1A, black). 
Interestingly, our triblock copolymers, PEO- $b$-PCL- $b$-PMOXA, with PMOXA length of 5 and 10 units (Fig. 1A, red and blue) also follow the literature trend for diblock copolymers. An exception is that PEO- $b$-PCL- $b$-PMOXA polymersomes have asymmetric membrane in contrast to PEO- $b$-PCL polymersomes as we have shown previously. ${ }^{16} \mathrm{We}$ observe a part of radically different trends for PEO- $b$-PCL- $b$-PMOXA with PMOXA length of 20 and 25 units (Fig. 1A, green and dark red). They consist of cloud-like aggregates (Fig. 1E, 2) that have not been reported previously for any amphiphilic block copolymer. These aggregates have loosely packed branched structure without any defined pattern and with dimensions up to few hundred $\mu \mathrm{m}$ (Fig. 2A, C). Typically, these structures coexist with polymersomes often found on the edges of the cloud-like aggregates (indicated by arrows on Fig. 2B, D). We discuss possible reason of the formation of cloud-like aggregates further in the text.

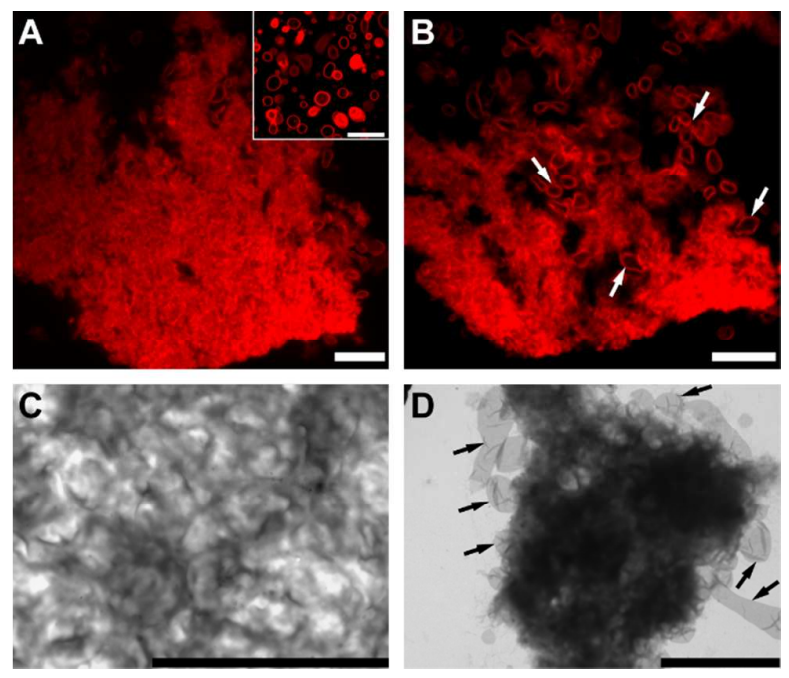

Figure 2. Characterization of cloud-like aggregates formed by $\mathrm{E}_{45} \mathrm{C}_{135} \mathrm{M}_{20}$ in aqueous solution. (A, B) LSM images at different z-focal planes: (A) middle; (B) lower surface. Inset in image $A$ represents polymersomes formed by this polymer $\left(\mathrm{E}_{45} \mathrm{C}_{135} \mathrm{M}_{20}\right)$ in PBS. Structures were stained with Bodipy 630/650. (C, D) TEM images of non-stained cloud-like aggregates. Arrows on images B and D point out polymersomes which are often found on the edges of cloud-like aggregates. Scale bars are $10 \mu \mathrm{m}$. 
So far, we have looked at morphology trends while increasing $f$ by decreasing PCL length and keeping PMOXA length constant. However, for PEO- $b$-PCL- $b$-PMOXA, $f$ can also be increased by increasing PMOXA length and keeping PCL length constant. Such flexibility in increasing $f$ is, obviously, due to an additional degree of freedom brought into the system by C (PMOXA) block. Since there is lack of systematic data for ABC polymers in literature, morphology trends are not known for the case when $f$ is varied through $\mathrm{C}$ block. We made a first attempt and revealed such (partial) morphology trends as discussed below.

In Fig. 1A, we split the tested polymers into 3 groups with common morphology trends: polymers with PCL $\sim 50$ units, polymers with PCL $\sim 60-130$ units, and polymers with PCL $\sim 150$ units. Polymers with the shortest PCL $\sim 50$ units formed predominantly spherical particles and partially dissolved under the tested conditions. In the case of polymers with PCL $\sim 60-130$ units morphology of the structures changes in the row spherical particles (Fig. 1B) - polymersomes (Fig. 1D) - cloud-like aggregates (Fig. 1E) with an increase in $f$. For polymers with PCL $\sim 150$ units with an increase in $f$ morphology of the structures changes from spherical particles/polymersomes to irregularly shaped particles (Fig. 1C). Irregularly shaped particles are observed along with macroscopic precipitate, and can be considered as smaller pieces of the insoluble polymer.

To explain formation of the observed self-assembled structures, we refer to packing geometry model. For AB diblock copolymers, packing geometry is defined by packing parameter $p:^{33}$

$$
p=\frac{v_{B}}{a_{A-B} \cdot l_{B}}
$$

where $a_{A-B}$ is the optimal area of the hydrophilic block $\mathrm{A}$ at the interface $\mathrm{A}-\mathrm{B}, v_{B}$ and $l_{B}$ are the volume and critical length of the hydrophobic block B, respectively.

PEO- $b$-PCL polymers with PCL $\sim 150$ units form polymersomes, and according to packing geometry model, individual molecules of these polymers are packed into cylinders 
(Scheme 2a). We speculate that addition of PMOXA block does not influence packing geometry for PEO-PCL interface, but adds another area $a_{C-B}$ and creates a new packing geometry at the C-B (PMOXA-PCL) interface (Scheme 2b). Such assumption is based on the immiscibility of PEO and PMOXA polymers in aqueous solution. ${ }^{34-35}$ The final packing geometry of PEO- $b$-PCL- $b$-PMOXA molecules can be considered as a result of the superposition of two packing geometries for PEO-PCL and PMOXA-PCL interfaces. PEO$b$-PCL- $b$-PMOXA with PCL $\sim 150$ units precipitated in FR method, in contrast to the corresponding PEO- $b$-PCL polymers which formed polymersomes and spherical particles. We attribute it to the appearance of PMOXA-PCL packing geometry which corresponds to precipitate. The combination of long PCL and relatively short PMOXA results in high curvature at PMOXA-PCL interface which prevents triblock copolymers from being dispersed, i.e. hydrophobic attractive forces between PCL chains become predominant over repulsive forces between PEO and PMOXA chains, and the triblock copolymer remains as a precipitate.

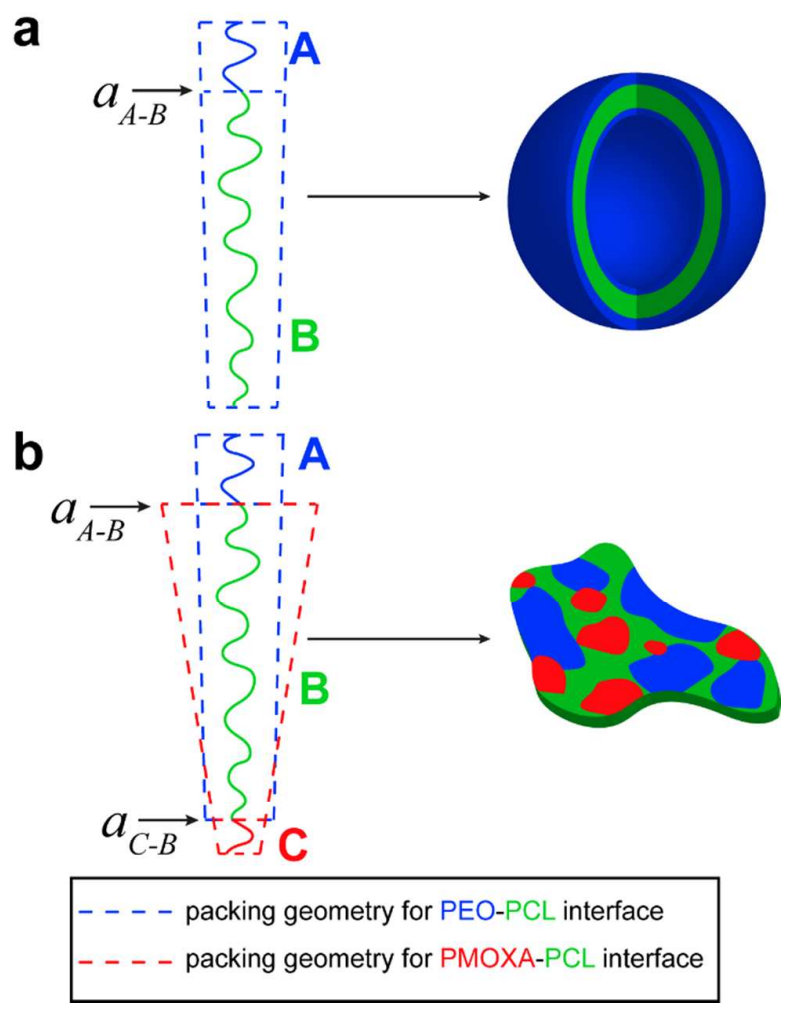


Scheme 2. Illustration of the packing geometry of polymers with PCL $\sim 150$ units: (a) AB and (b) $\mathrm{ABC}$ resulting into polymersomes and irregularly shaped particles (precipitate), respectively.

Morphology of the self-assembled structures formed by polymers with PCL $\sim 60-130$ units changes in the row spherical particles $(\mathrm{PMOXA}=0$ units $)-$ polymersomes $(\mathrm{PMOXA}=$ 5-10 units, Fig. 1D) - cloud-like aggregates (PMOXA = 20-25 units, Fig. 1E, 2) with an increase in $f$. In this case, change of PCL length ( $~ 60-130$ units) has almost no effect on self-assembled morphology presumably due to the ability of PCL chains to compress or stretch in the fluid state (for PCL above $\mathrm{T}_{\mathrm{m}}$ ), which leads to the adjustment of a polymer molecule to a certain packing geometry. ${ }^{33}$ PEO- $b$-PCL form spherical particles composed of distinct domains, and such domains possess shapes ranging from spheres to rods (Fig. 1B inset, S3). Despite the mechanism of self-assembly of such particles remains unclear, the packing geometry of PEO- $b$-PCL molecules can be attributed to a cone (spherical micelles) and truncated cylinder (rod-like micelles). For simplicity reasons, we generalize packing geometry of PEO- $b$-PCL forming spherical particles as a cone (Scheme 3a). 

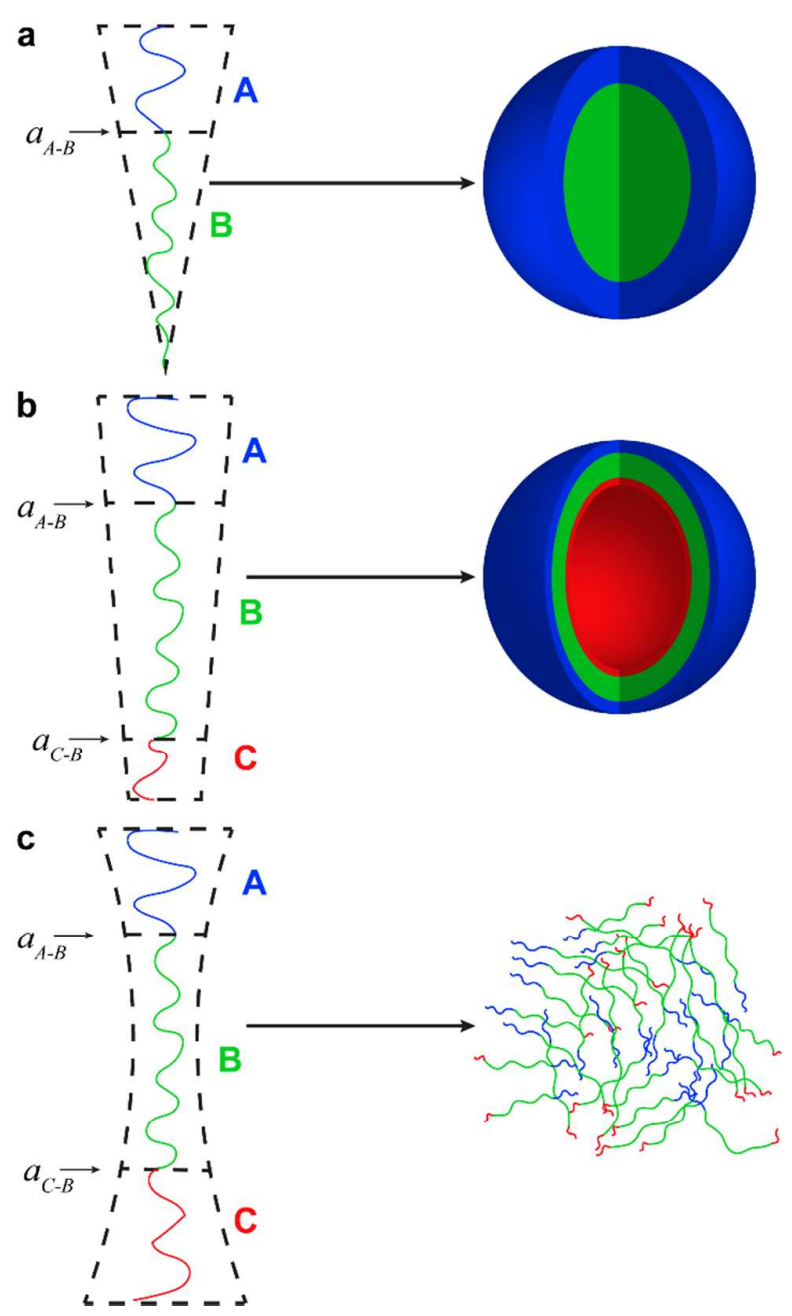

Scheme 3. Illustration of the packing geometry of polymers with fixed A and B $(\sim 60-130$ units) but different $\mathrm{C}$ block lengths: (a) $\mathrm{AB}$, (b) $\mathrm{ABC}$ with short $\mathrm{C}$ block, and (c) $\mathrm{ABC}$ with long C block.

PEO- $b$-PCL- $b$-PMOXA polymers with PCL $\sim 60-130$ and relatively short PMOXA (5-10 units) form polymersomes with diameters $\sim 3 \mu \mathrm{m}$ (Fig. 1D, Table 1). According to the Student's t-test ${ }^{36}$ with $\mathrm{p}=0.05$, the values of diameters are not significantly different. As discussed above, addition of PMOXA block presumably introduces area $a_{C-B}$ at the $\mathrm{C}-\mathrm{B}$ (PMOXA-PCL) interface. Assuming that hydrophilic blocks are in the stretched conformation, the counter length of PEO (162.00 $\AA$ ) is longer than that of PMOXA (for 5/10 units the length is $18.30 / 36.60 \AA$ ) (for calculations see Supporting Information). The 
approximate diameter of PEO chain $(\sim 2 \AA)$ is smaller compared to that of PMOXA $(\sim 7 \AA)$ due to the side chain in PMOXA backbone, and this difference is likely to be larger in the hydrated state, since PEO needs 3 water molecules ${ }^{37}$ and PMOXA 5.2 per repeating unit. ${ }^{35}$ Nevertheless, due to much longer PEO length the resulting packing geometry of $\mathrm{ABC}$ molecules corresponds to a cylinder slightly truncated at the $\mathrm{C}$ side (Scheme $3 \mathrm{~b}$ ). Such packing geometry results in polymersomes with the inner surface formed by a shorter PMOXA block, and the outer surface formed by a longer PEO block (Scheme 3b), which we indeed proved by two independent methods. ${ }^{16}$

Table 1. Average diameter of polymersomes formed by different polymers in film rehydration method. The diameter was determined based on at least 3 different LSM images containing at least 300 objects in total.

\begin{tabular}{|c|c|}
\hline Copolymer & Polymersome diameter, $\boldsymbol{\mu m}$ \\
\hline $\mathrm{E}_{45} \mathrm{C}_{66} \mathrm{M}_{5}$ & $2.4 \pm 0.9$ \\
\hline $\mathrm{E}_{45} \mathrm{C}_{103} \mathrm{M}_{4}$ & $2.8 \pm 1.2$ \\
\hline $\mathrm{E}_{45} \mathrm{C}_{103} \mathrm{M}_{12}$ & $3.1 \pm 1.2$ \\
\hline $\mathrm{E}_{45} \mathrm{C}_{110} \mathrm{M}_{4}$ & $3.1 \pm 1.2$ \\
\hline $\mathrm{E}_{45} \mathrm{C}_{135} \mathrm{M}_{4}$ & $3.5 \pm 1.4$ \\
\hline $\mathrm{E}_{45} \mathrm{C}_{135} \mathrm{M}_{10}$ & $3.2 \pm 1.3$ \\
\hline $\mathrm{E}_{45} \mathrm{C}_{153}$ & $1.3 \pm 0.5$ \\
\hline
\end{tabular}

PEO- $b$-PCL- $b$-PMOXA polymers with PCL $\sim 60-130$ and relatively long PMOXA (20-25 units) self-assembled into cloud-like aggregates (Fig. 1E, 2). We explain formation of such morphology by the increase of $a_{C-B}$ interfacial area compared to PEO- $b$-PCL- $b$-PMOXA polymers with a shorter PMOXA (5-10 units) (Scheme 3c). In the case of PMOXA 20/25 units its larger length (73.20/91.50 $\AA$ for $20 / 25$ units $)$ and diameter $(\sim 7 \AA+5.2$ water 
molecules per repeating unit $^{35}$ ) results in the increase of repulsive forces between hydrophilic chains which compete with hydrophobic attractive forces between PCL chains. The packing geometry of $\mathrm{ABC}$ molecules in this case approaches the double cone shape (Scheme $3 \mathrm{c}$ ). The aggregates have 3D-extended structure of several hundred $\mu \mathrm{m}$ size. Such branched structure with large size supposedly helps shielding hydrophobic interactions from aqueous environment. To support our assumption that cloud-like aggregates form due to strong hydrophilic repulsion between water-soluble chains, we tried to decrease these forces by performing self-assembly in the presence of salts. In PBS buffer such polymers form predominately polymersomes (Fig. 2A, inset), suggesting that repulsion between hydrophilic chains indeed decreases in the presence of salts, and hence the packing geometry changes from double cone shape (Scheme 3c) to cylinder (Scheme 3b).

Solvent evaporation method. In solvent evaporation method, a polymer was dissolved in $\mathrm{CH}_{2} \mathrm{Cl}_{2}$ in a glass vial. After addition of water the solution was stirred at $22{ }^{\circ} \mathrm{C}$ in the open vial until $\mathrm{CH}_{2} \mathrm{Cl}_{2}$ evaporated. Fig. 3A summarizes the predominant structures formed by PEO- $b$-PCL- $b$-PMOXA and their precursors, PEO- $b$-PCL, in solvent evaporation method. Similar to film rehydration method, most of polymers formed two or three types of structures, but to simplify the morphology diagram in Fig. 3A, only predominant structures are plotted. More detailed information on morphology of the self-assembled structures can be found in Table S1. 

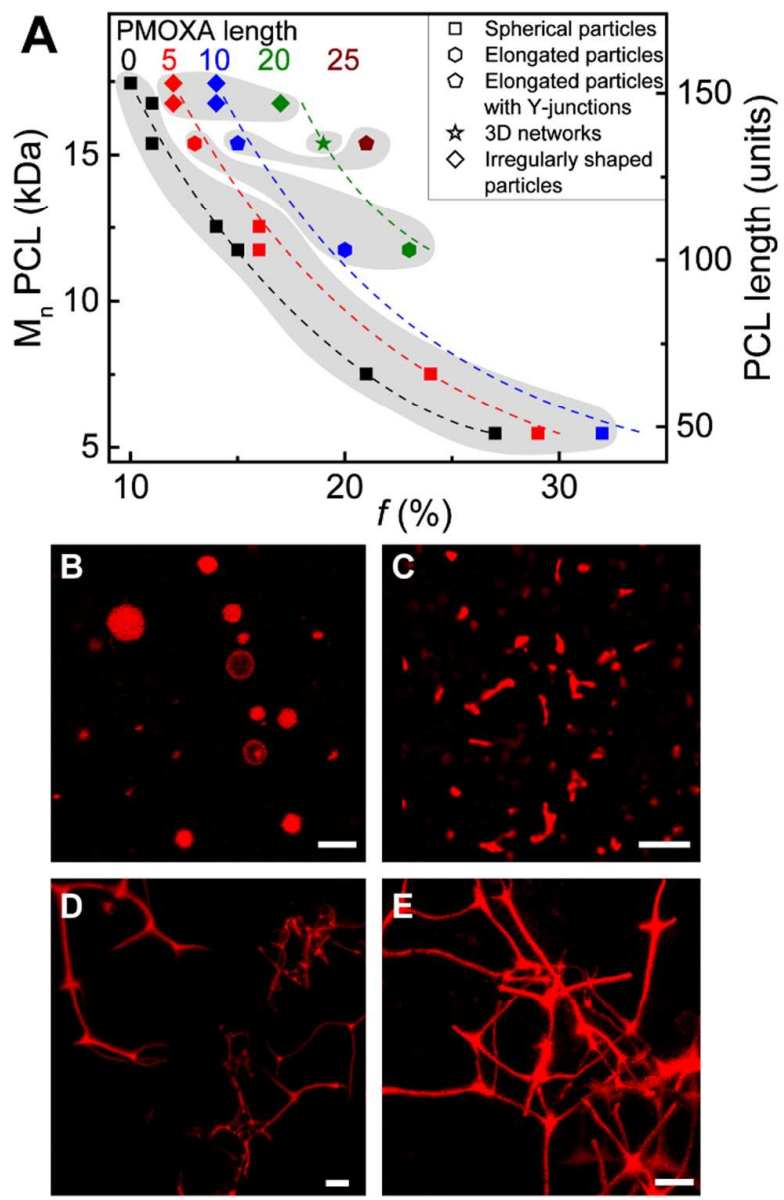

Figure 3. Self-assembled structures observed in solvent evaporation method. (A) Morphology diagram showing structures formed by PEO- $b$-PCL and PEO- $b$-PCL- $b$-PMOXA in aqueous solution as a function of the molecular composition. Points of each color correspond to polymers with a certain PMOXA length. Points of each shape correspond to a certain morphology: spherical particles (squares), elongated structures (hexagons), elongated structures with Y-junctions (pentagons), 3D networks (star), irregularly shaped particles (diamonds). The gray areas point out regions of the same morphology. Representative LSM images of the structures formed by (B) $\mathrm{E}_{45} \mathrm{C}_{153}$ - spherical particles, (C) $\mathrm{E}_{45} \mathrm{C}_{103} \mathrm{M}_{12}-$ elongated particles. z-projection of z-stack CLSM of the structures formed by (D) $\mathrm{E}_{45} \mathrm{C}_{135} \mathrm{M}_{10}$ - elongated particles with $\mathrm{Y}$-junctions and (E) $\mathrm{E}_{45} \mathrm{C}_{135} \mathrm{M}_{20}-3 \mathrm{D}$ networks. Structures were stained with Bodipy 630/650. Scale bars are $5 \mu \mathrm{m}$. 
In the absence of PMOXA block, i.e. in the case of PEO- $b$-PCL, self-assembled structures are predominantly spherical particles with diameters $0.2-2 \mu \mathrm{m}$ (Fig. 3B; the range of diameters was determined from TEM). In the case of PEO- $b$-PCL- $b$-PMOXA with PMOXA 5, morphology of the structures changes in the row irregularly shaped particles elongated particles - spherical particles with an increase in $f$. Morphology of the structures formed by PEO- $b$-PCL- $b$-PMOXA with PMOXA 10 undergoes transition irregularly shaped particles - elongated particles with Y-junctions (Fig. 3D) - elongated particles (Fig. 3C) spherical particles with an increase in $f$. In the case of PEO- $b$-PCL- $b$-PMOXA with PMOXA length of 20 units, morphology of the self-assembled structures changes in the row irregularly shaped particles - 3D networks (Fig. 3E) - elongated particles with an increase in $f$. Typical literature trend for PEO- $b$-PCL assembled under similar solvent evaporation conditions is irregularly shaped particles - polymersomes - spherical particles with increasing $f .{ }^{38}$ Our morphology trends for PEO- $b$-PCL and PEO- $b$-PCL- $b$-PMOXA differ dramatically: we did not observe polymersomes as a predominant morphology. Interestingly, few PEO- $b$-PCL ${ }^{38}$ polymers were reported to self-assemble into elongated structures, whereas our PEO- $b$-PCL with comparable molecular composition formed spherical particles.

To reveal morphology trends while $f$ is varied through $\mathrm{C}$ block, we divide the tested polymers into 4 groups in Fig. 3A: polymers with PCL $\sim 50-70$ units, polymers with PCL $\sim 100$ units, polymers with PCL $\sim 130$ units, and polymers with PCL $\sim 150$ units. Polymers with the shortest PCL $\sim 50-70$ units formed predominantly spherical particles. Morphology of the self-assembled structures formed by polymers with PCL $\sim 100$ units changes from spherical particles to elongated particles (Fig. 3C) while increasing $f$. In the case of polymers with PCL $\sim 130$ units morphology of the structures changes in the row spherical particles elongated particles - elongated particles with Y-junctions (Fig. 3D) - 3D networks (Fig. 3E) - elongated particles with Y-junctions with an increase in $f$. Morphology of the self- 
assembled structures formed by polymers with PCL $\sim 150$ units changes from spherical particles to irregularly shaped particles.

From the trends discussed above one can observe some similarities between film rehydration and solvent evaporation methods: PEO- $b$-PCL form predominantly spherical particles; PEO- $b$-PCL- $b$-PMOXA with PCL $\sim 50$ units and $\sim 150$ units form spherical particles and irregularly shaped particles, respectively. These findings suggest little effect of the preparation method on the self-assembly of these particular polymers. On the other hand, PEO- $b$-PCL- $b$-PMOXA polymers with PCL $\sim 60-130$ units self-assemble into elongated rod-like structures in solvent evaporation method, while forming polymersomes and cloudlike aggregates in film rehydration method.

PEO- $b$-PCL- $b$-PMOXA polymers with PCL $\sim 130$ units form rods with Y-junctions and 3D microscale networks. Formation of such structures by amphiphilic block copolymers is rarely observed and so far was reported only for few AB systems. ${ }^{8,}{ }^{39}$ It is believed that such networks are formed above a critical molecular weight of the polymer. ${ }^{8}$ Similarly to poly(ethylene oxide)-block-polybutadiene (PEO-b-PB) 3D nanoscale networks, ${ }^{8}$ we also observed macroscopic phase separation in the case of PEO- $b$-PCL- $b$-PMOXA 3D microscale networks. To gain insight into the microscale structure of these networks, we characterized them using z-stack CLSM (Fig. 4A). Interestingly, the polymer forming these networks, $\mathrm{E}_{45} \mathrm{C}_{135} \mathrm{M}_{20}$, assembles into $3 \mathrm{D}$ cloud-like aggregates in film rehydration method (Fig. $4 \mathrm{~A}$ inset, 1E, 2), suggesting the inherent tendency of this polymer to form branched 3D structures. 3D networks are composed of rods typically with a length of 5-50 $\mu \mathrm{m}$ and thickness up to $10 \mu \mathrm{m}$. To gain a better understanding of the structure of the networks at the nanoscale, we performed TEM (Fig. 4B-E) and cryoTEM (Fig. 4F-J) imaging. Since these techniques require a special sample preparation procedure, most of the microscale parts of $3 \mathrm{D}$ networks were blotted off. We found only one piece of networks in the negatively stained 
TEM sample (Fig. 4B). The thickest rods of the networks might have porous structure (Fig. $4 \mathrm{~B}$, inset). Some of the thinner rods with diameters $\sim 20-200 \mathrm{~nm}$ possibly exhibit hollow structure (pointed out by arrows on Fig. 4C, D, G, H), but this cannot be undoubtedly concluded. In some TEM and cryoTEM images we observed rods which have a helical structure or are composed of the disks stacked together (Fig. 4E, I, J), and diameter of these rods ranges from $\sim 20$ up to $\sim 500 \mathrm{~nm}$.

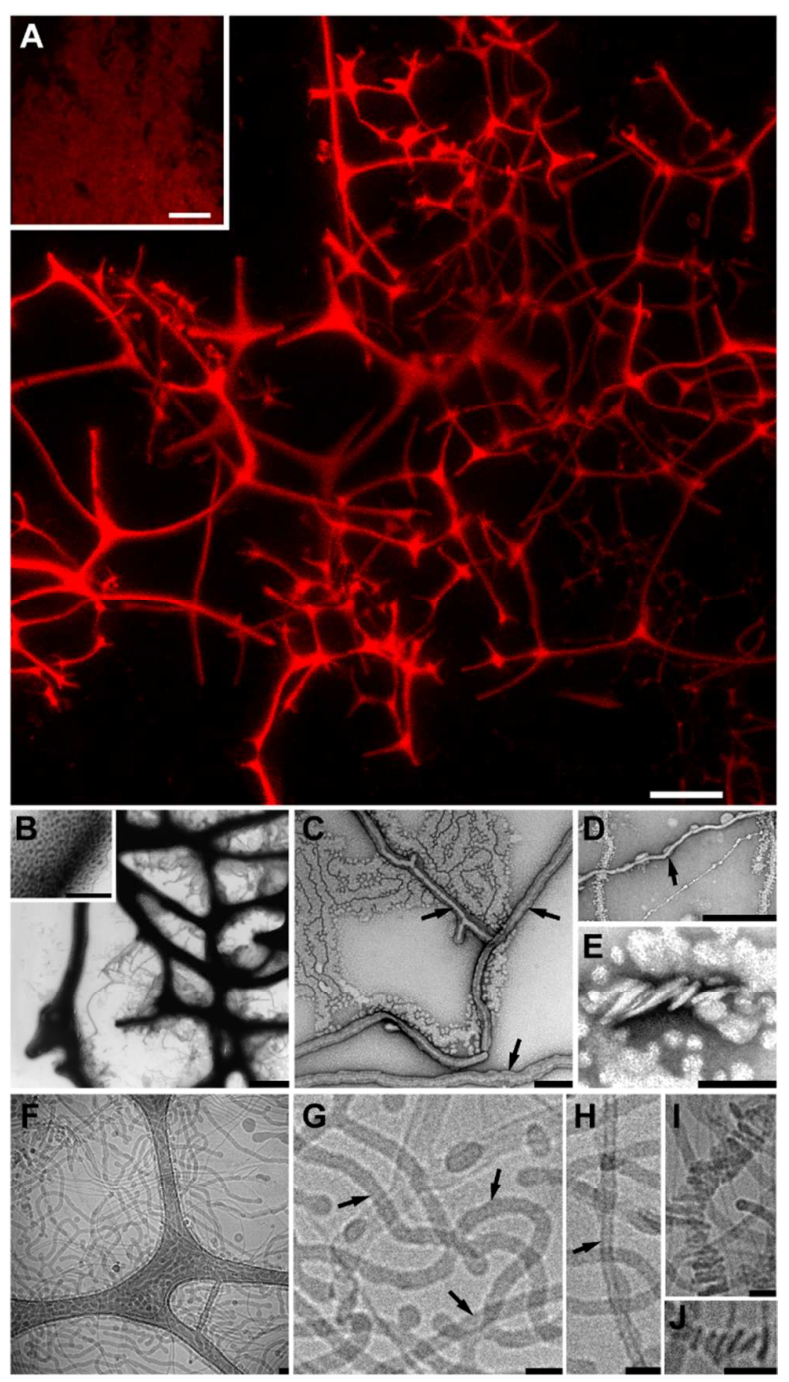

Figure 4. Characterization of $3 \mathrm{D}$ networks formed by $\mathrm{E}_{45} \mathrm{C}_{135} \mathrm{M}_{20}$ in solvent evaporation method: (A) z-projection of z-stack CLSM, inset: cloud-like aggregates formed by the same polymer in film rehydration method (LSM); structures were stained with Bodipy 630/650. 
(B-E) negatively stained TEM; (F-J) cryoTEM. Scale bars: A, A inset, B $10 \mu \mathrm{m}$; B inset, C-E $1 \mu \mathrm{m}$; F-J $50 \mathrm{~nm}$. The arrows on C, D, G, and H point out possibly hollow rods.

Formation of the self-assembled structures observed in solvent evaporation method can be explained in the following way. Amphiphilic polymers dissolved in $\mathrm{CH}_{2} \mathrm{Cl}_{2}$ upon addition of water and stirring start to orient with their hydrophilic tails towards aqueous solution (Scheme 4). Diblock copolymers have only one hydrophilic tail, and therefore they form mainly I-shaped monolayers at the $\mathrm{CH}_{2} \mathrm{Cl}_{2}-\mathrm{H}_{2} \mathrm{O}$ interface. Upon evaporation of the organic solvent, these monolayers transfer into aqueous solution in the form of spherical particles (Scheme 4a). Triblock copolymers have two water-soluble blocks, A and C, and both of them tend to transfer into water phase, thus resulting in U-shaped loops at the $\mathrm{CH}_{2} \mathrm{Cl}_{2}-\mathrm{H}_{2} \mathrm{O}$ interface. When $\mathrm{C}$ block is relatively short, its attraction into water phase is weaker than that of PEO (i.e. $5.2 \times 5=26$ water molecules for PMOXA ${ }^{35} 5$ units vs. $3 \times 45=$ 135 water molecules for $\mathrm{PEO}^{37}$ ). Triblock copolymers form a mixture of I-shaped monolayers and U-shaped loops, which upon evaporation of $\mathrm{CH}_{2} \mathrm{Cl}_{2}$ transfer into spherical particles and short rods (Scheme $4 b$ ). In the case of relatively long $\mathrm{C}$ block, its attraction into water phase becomes stronger (i.e. 52 water molecules for PMOXA 10 units vs. 135 for $\mathrm{PEO}$ ), and $\mathrm{ABC}$ molecules predominantly form loops at the $\mathrm{CH}_{2} \mathrm{Cl}_{2}-\mathrm{H}_{2} \mathrm{O}$ interface. Upon evaporation of the organic solvent, these loops transfer into aqueous solution in the form of long rods (Scheme $4 \mathrm{c})$. The microscale rods with thickness $>140 \mathrm{~nm}(2 \times$ length of triblock copolymer in U-shaped confirmation, see Supporting Information) might possess hollow morphology, but as has been shown for 3D networks (Fig. 4), this cannot be undoubtedly concluded from LSM images due to limited resolution and from TEM or cryoTEM analysis due to sample preparation procedure leading to removal of thick rods.

Thus, spherical particles are observed for PEO- $b$-PCL polymers (Fig. 2B), spherical and short elongated $(<5 \mu \mathrm{m})$ particles are common for PEO- $b$-PCL- $b$-PMOXA polymers 
with short PMOXA ( $\sim 5$ units), and mostly long $(>5 \mu \mathrm{m})$ elongated structures are observed for PEO- $b$-PCL- $b$-PMOXA with longer PMOXA (> 5 units) (Fig. 3C-E, 4, Table 2). Size values of the elongated structures scattered too much and could not be fitted with conventional functions for statistical analysis, therefore only the range of the lengths is provided in Table 2.
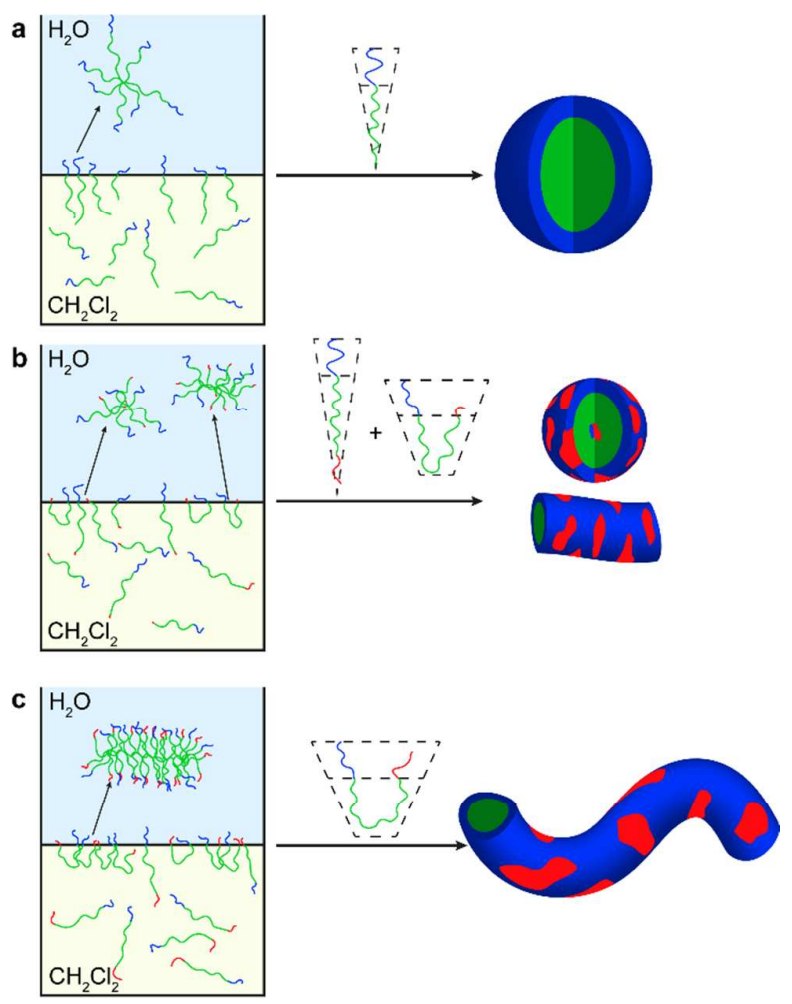

Scheme 4. Proposed mechanism of self-assembly in solvent evaporation method for: (a) AB polymers, (b) $\mathrm{ABC}$ with short $\mathrm{C}$, (c) ABC with long $\mathrm{C}$.

Table 2. Length of elongated structures formed by different PEO- $b$-PCL- $b$-PMOXA polymers in solvent evaporation method. The length was determined based on at least 3 different images containing at least 70 objects in total.

\begin{tabular}{|c|c|}
\hline Copolymer & Range of the lengths of elongated structures, $\boldsymbol{\mu m}$ \\
\hline $\mathrm{E}_{45} \mathrm{C}_{48} \mathrm{M}_{3}$ & $1.5-4.5$ \\
\hline $\mathrm{E}_{45} \mathrm{C}_{66} \mathrm{M}_{5}$ & $1.5-5$ \\
\hline
\end{tabular}




\begin{tabular}{|c|c|}
\hline $\mathrm{E}_{45} \mathrm{C}_{110} \mathrm{M}_{4}$ & $1-6$ \\
\hline $\mathrm{E}_{45} \mathrm{C}_{103} \mathrm{M}_{12}$ & $5-40$ \\
\hline $\mathrm{E}_{45} \mathrm{C}_{135} \mathrm{M}_{10}$ & $5-30$ \\
\hline $\mathrm{E}_{45} \mathrm{C}_{135} \mathrm{M}_{25}$ & $2-40$ \\
\hline
\end{tabular}

For PEO- $b$-PCL ${ }^{38}$ and PEO- $b$-PCL- $b$-PMOXA elongated structures are uniquely accessible only via solvent evaporation method. As discussed above, we attribute formation of elongated structures to the presence of two terminal hydrophilic blocks. To prove the latter assumption, we compared self-assembly of diblock copolymer $\mathrm{E}_{45} \mathrm{C}_{153}$ (PEO(2.0K)- $b$ $\left.\operatorname{PCL}(17.4 \mathrm{~K}), \mathrm{Ð}_{\mathrm{M}}=1.08\right)$ and its analogue $\mathrm{PEO}(2.0 \mathrm{~K})-b-\mathrm{PCL}(16.0 \mathrm{~K})\left(\bigoplus_{\mathrm{M}}=1.23\right)$ containing $26 \%$ of PEO- $b$-PCL- $b$-PEO species. ${ }^{31} \mathrm{E}_{45} \mathrm{C}_{153}$ assembled mainly into spherical particles, whereas polymer PEO(2.0K)-b-PCL(16.0K) formed mostly elongated and irregularly shaped structures in solvent evaporation method (Fig. S4). These findings support the proposed mechanism of self-assembly of di- and triblock copolymers in solvent evaporation method and also possibly explain how PEO- $b-\mathrm{PCL}^{38}$ formed elongated structures under similar conditions, since those polymers most likely contained some PEO- $b$-PCL- $b$-PEO species. ${ }^{31}$

Co-solvent method. In co-solvent method, a polymer was dissolved in THF, and the resulting solution was added dropwise into a glass vial with stirred aqueous solution. The mixture was stirred at $22{ }^{\circ} \mathrm{C}$ in the closed vial. In this method, we tested only polymers with PCL 110 - 153 units, because polymers already with PCL 110 units dissolved under these conditions.

Fig. 5A depicts the predominant structures formed by PEO- $b$-PCL- $b$-PMOXA and their precursors, PEO- $b$-PCL, in co-solvent method. Similar to film rehydration and solvent evaporation methods, most of the polymers formed two or three types of structures, but to simplify the morphology diagram in Fig. 5A, only predominant structures are plotted. More 
detailed information on morphology of the self-assembled structures can be found in Table S1.
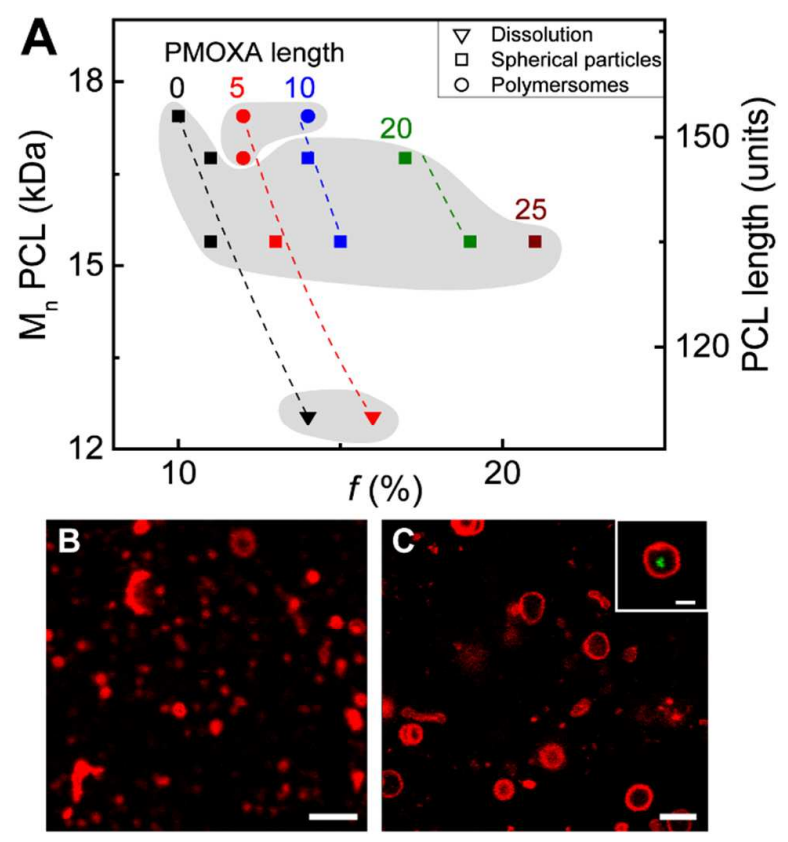

Figure 5. Self-assembled structures observed in co-solvent method. (A) Morphology diagram showing structures formed by PEO- $b$-PCL and PEO- $b$-PCL- $b$-PMOXA in aqueous solution as a function of the molecular composition. Points of each color correspond to polymers with a certain PMOXA length. Points of each shape correspond to a certain morphological state: dissolution (triangles), spherical particles (squares), polymersomes (circles). The gray areas point out regions of the same morphology. Representative LSM images of the structures formed by (B) $\mathrm{E}_{45} \mathrm{C}_{135} \mathrm{M}_{10}$ - spherical particles, (C) $\mathrm{E}_{45} \mathrm{C}_{153} \mathrm{M}_{11}$ - polymersomes. Structures were stained with Bodipy 630/650. Scale bars are $5 \mu \mathrm{m}$. Inset in the image (C) represents polymersomes with encapsulated hydrophilic dye calcein; scale bar is $2 \mu \mathrm{m}$.

PEO- $b$-PCL, i.e. where PMOXA length is 0 , self-assembled predominantly into spherical particles with diameters $0.2-1.5 \mu \mathrm{m}$. In the case of PEO- $b$-PCL- $b$-PMOXA with PMOXA 5 and 10 units, morphology of the self-assembled structures changes from polymersomes to spherical particles (Fig. 5B), and the polymers then dissolve with an 
increase in $f$. PEO- $b$-PCL- $b$-PMOXA with PMOXA 20 and 25 units self-assembled into spherical particles.

Morphology trends when $f$ is varied through $\mathrm{C}$ block are the following. Polymers with PCL 110 units predominantly dissolved in co-solvent method. The diblock copolymer formed some spherical particles, whereas the triblock copolymer dissolved completely and resulted in optically transparent solution. Such self-assembly behavior of the triblock copolymer can be attributed to the weakening of hydrophobic interactions between PCL chains in the presence of THF which are not able to compensate hydrophilic repulsive forces in the corona. Polymers with PCL 135 units self-assembled into spherical particles (Fig. 5B), which is analogous to the case of PCL $\sim 50$ units in FR method. Morphology of the structures formed by polymers with PCL 147 changes in the row spherical particles - polymersomes - spherical particles while increasing $f$. For polymers with PCL 153 units, with an increase in $f$ morphology undergoes transition from spherical particles to polymersomes (Fig. 5C), which is analogous to the trend observed for PCL $\sim 60-130$ units in FR method. The average diameter of polymersomes formed in co-solvent method is $\sim 3 \mu \mathrm{m}$ (Table 3) which is similar to the ones formed in film rehydration method (Table 1).

Table 3. Average diameter of polymersomes formed by PEO- $b$-PCL- $b$-PMOXA polymers in co-solvent method. The diameter was determined based on at least 3 different LSM images containing at least 300 objects in total.

\begin{tabular}{|c|c|}
\hline Copolymer & Polymersome diameter, $\boldsymbol{\mu m}$ \\
\hline $\mathrm{E}_{45} \mathrm{C}_{147} \mathrm{M}_{4}$ & $2.4 \pm 0.6$ \\
\hline $\mathrm{E}_{45} \mathrm{C}_{153} \mathrm{M}_{4}$ & $2.5 \pm 0.8$ \\
\hline $\mathrm{E}_{45} \mathrm{C}_{153} \mathrm{M}_{11}$ & $3.3 \pm 0.9$ \\
\hline
\end{tabular}

To the best of our knowledge, there are no studies reporting about systematic investigation of self-assembly of PEO-b-PCL in co-solvent method. In the case of our 
polymers, co-solvent method allowed us to expand the range of the self-assembled structures for polymers which precipitated in film rehydration and solvent evaporation methods. The key difference of co-solvent method is the presence of $20 \%$ THF in the self-assembly mixture. Considering packing geometry model, we assume that this solvent composition does not change interactions between PEO chains, because this block is soluble in both water and THF. THF is a good solvent for PCL block, and therefore $\mathrm{THF}-\mathrm{H}_{2} \mathrm{O}$ mixture is able to solubilize partially PCL block. Thus, the effective $v_{B}$ and $l_{B}$ occupied by the PCL block changes leading to different self-assembly behavior of PEO- $b$-PCL polymers compared to film rehydration and solvent evaporation methods (Scheme 5a). For example, while forming predominantly polymersomes in film rehydration method, $\mathrm{E}_{45} \mathrm{C}_{153}$ self-assembled into spherical particles and partially dissolved in co-solvent method. The presence of $20 \%$ THF has similar effect in the case of PEO- $b$-PCL- $b$-PMOXA insoluble in film rehydration and solvent evaporation methods, but the self-assembled morphology changes from precipitate to polymersomes (Scheme 5b). 


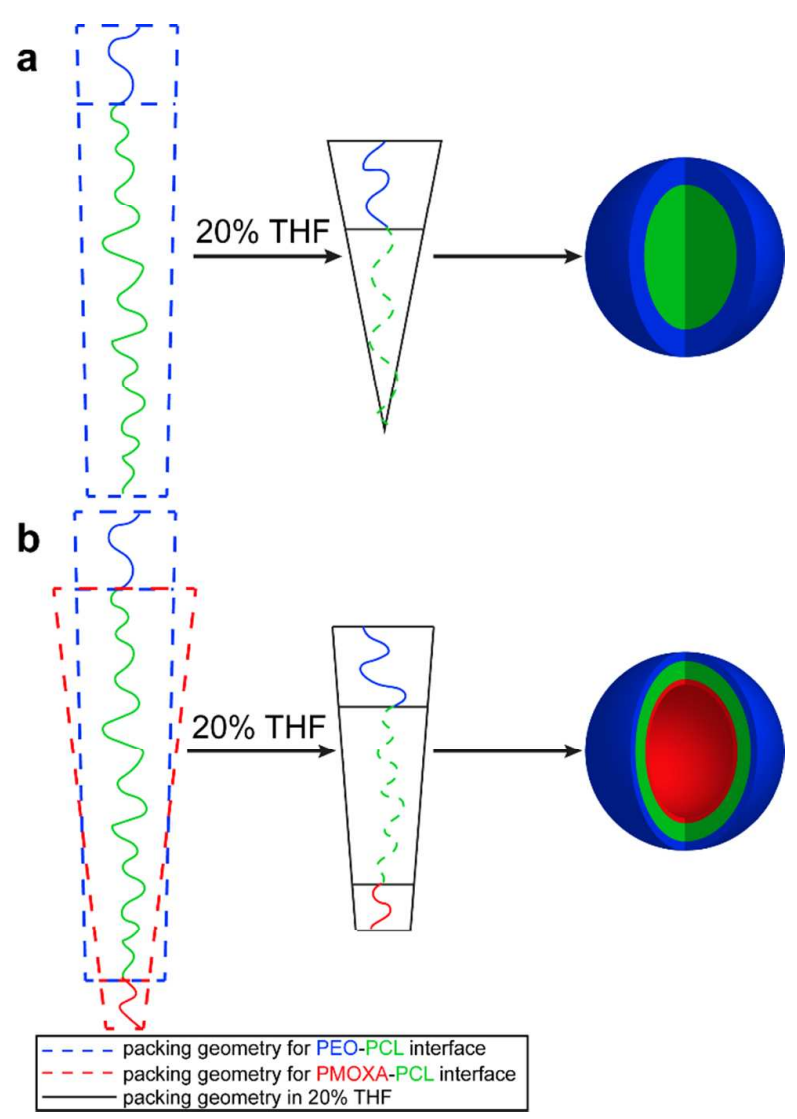

Scheme 5. Illustration how packing geometry of (a) AB and (b) ABC polymers changes in the presence of $20 \%$ THF.

The morphological trend observed for polymers with PCL 147 (spherical particles polymersomes - spherical particles) is not completely clear, but it might deal with partial solubility of PMOXA in THF- $\mathrm{H}_{2} \mathrm{O}$ mixture. PMOXA homopolymer precipitates in THF. In the case of $\mathrm{E}_{45} \mathrm{C}_{147} \mathrm{M}_{4}$ formation of polymersomes can be explained as described above (Scheme 5b). With further increase of PMOXA length its insolubility in $\mathrm{THF}-\mathrm{H}_{2} \mathrm{O}$ mixture becomes more pronounced, and probably PMOXA block tends to collapse resulting in structures with only one completely soluble block - PEO.

As has been shown for some amphiphilic diblock copolymers, ${ }^{11,40-41}$ nature of a cosolvent (i.e. a common solvent for both blocks) has a dramatic effect on the observed morphology. To check whether a nature of the co-solvent affects self-assembly of PEO- $b$ PCL- $b$-PMOXA polymers, we tested the self-assembly of $\mathrm{E}_{45} \mathrm{C}_{153} \mathrm{M}_{4}$ using different co- 
solvents: DMF, ACN, and acetone. These solvents are among very few ones able to dissolve our triblock copolymers. The change of the co-solvent led to the formation of completely different aggregates which were not observed when THF was used as a co-solvent (Fig. 6, S5). On the other hand, the assemblies obtained in the presence of DMF or ACN or acetone possessed quite similar structures with three-dimensionality. These observations can be explained by only partial solubility of PCL block in these solvents, whereas THF dissolves PCL completely, ${ }^{42}$ suggesting its unique role in the self-assembly behavior of PEO- $b$-PCL- $b$ PMOXA polymers.

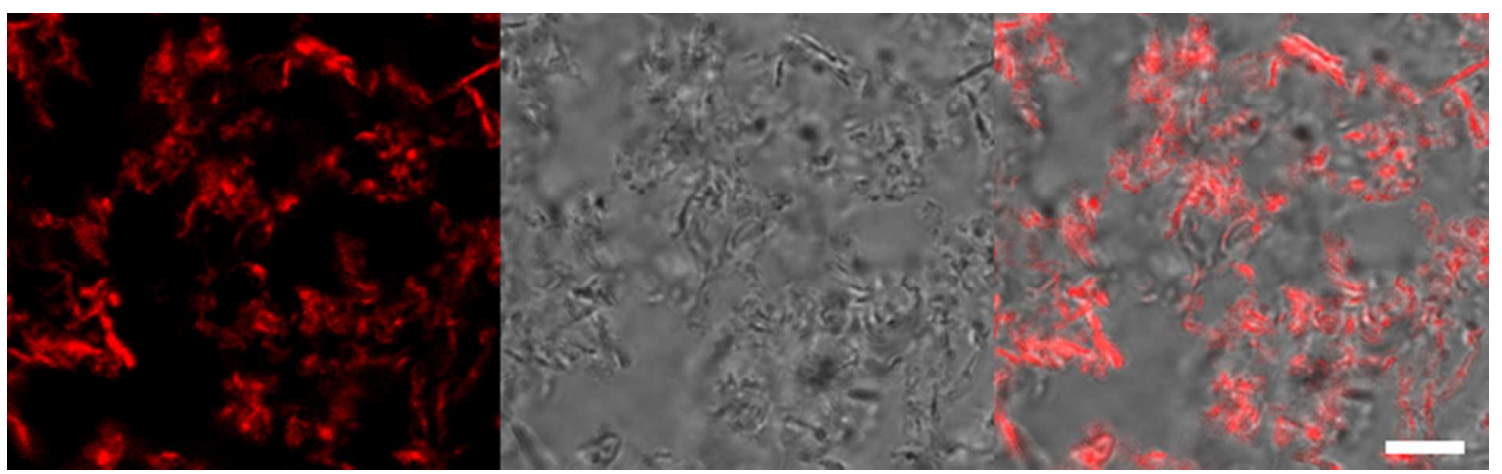

Figure 6. From left to right: LSM, bright field, and their overlay images of the aggregates formed by $\mathrm{E}_{45} \mathrm{C}_{153} \mathrm{M}_{4}$ obtained using acetone as a co-solvent. Structure was stained with Bodipy 630/650. Scale bar is $10 \mu \mathrm{m}$.

Equilibrium or kinetically frozen morphologies? Formation of diverse structures by PEO- $b$-PCL- $b$-PMOXA in different self-assembly methods suggests that one or all methods result in morphologies which are under kinetic control. Thermodynamically controlled structures should be insensitive to details in preparation procedure, whereas kinetically controlled morphologies are highly path dependent. ${ }^{6-7}$ Therefore, to gain a better understanding about self-assembly equilibrium, we studied how change of details in film rehydration, solvent evaporation, and co-solvent methods influences self-assembly. For the latter purpose, we chose polymers $\mathrm{E}_{45} \mathrm{C}_{110} \mathrm{M}_{4}, \mathrm{E}_{45} \mathrm{C}_{135} \mathrm{M}_{4}, \mathrm{E}_{45} \mathrm{C}_{135} \mathrm{M}_{20}$ as they have different PCL and PMOXA lengths and self-assemble into different microscale structures. In film 
rehydration method stirring rate (300-800 rpm), duration of self-assembly (6-48 h), and conditions of polymer film formation did not influence the self-assembled morphology of the selected polymers. Employment of different heating and cooling conditions for self-assembly also did not affect the type and size of the structures (Fig. 7, S6). These findings suggest that the structures formed in film rehydration method are energetically favorable and the system is on the way to approach thermodynamic equilibrium. The diameter values of polymersomes possessed high deviation $(\sim 40 \%)$ from the mean diameter value (Table 1$)$. This deviation can be partially attributed to the dispersity of the polymers and the presence of $\sim 15 \%$ of high molecular weight impurities. ${ }^{16} \mathrm{We}$ assume, however, that the main reason for this high deviation, as well as for the co-existence of multiple morphologies (Table S1, Fig. S2), is the fact that the system does not fully achieve global equilibrium on the experimental timescale (24 h), which is associated with hindered structural evolution due to slow kinetics of high molecular weight polymers. ${ }^{7,43}$ Longer experimental times $(>24 \mathrm{~h})$ are problematic because of high temperature $\left(62^{\circ} \mathrm{C}\right)$ : water evaporates from the solution containing self-assembled structures and condenses on the walls of the flask above the solution. Further in the text, we refer to the structures formed in film rehydration method as pseudoequilibrium morphologies, because the system might be on the way in achieving global equilibrium in this method.
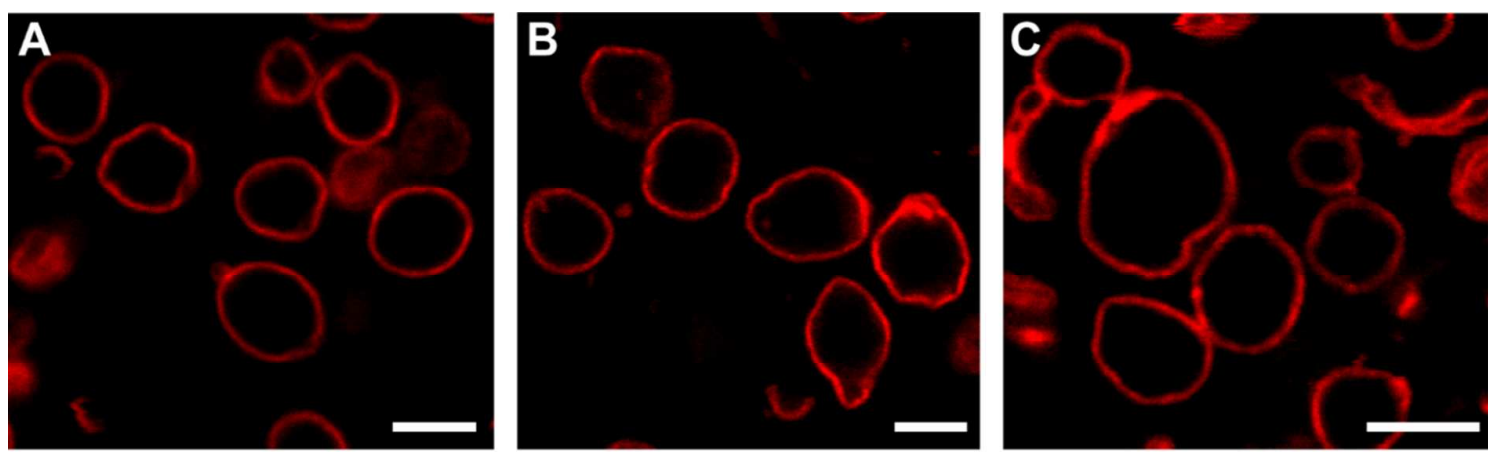

Figure 7. LSM images of the polymersomes formed by $\mathrm{E}_{45} \mathrm{C}_{110} \mathrm{M}_{4}$ obtained using film rehydration method under different cooling/heating conditions: (A) standard procedure; (B) 
slow heating; (C) slow cooling. Structures were stained with Bodipy 630/650. Scale bars are $5 \mu \mathrm{m}$.

As has been shown for most of $\mathrm{AB}^{8,44-46}$ and $\mathrm{ABC}^{30}$ systems in A-selective solvent, the type of morphology usually changes in the row polymersomes - elongated micelles spherical micelles with the increase of $f$. Assemblies formed by PEO- $b$-PCL ${ }^{32}$ and PEO- $b$ PCL- $b$-PMOXA are exceptions from this rule. These polymers do not form elongated micelles in film rehydration method and self-assemble into microscale structures, whereas most of the systems result in nanoscale assemblies. These observations are associated with the semicrystalline nature of PCL block and can be possibly attributed to the formation of PCL spherulites in the bulk phase,$^{47}$ which might affect formation of the core of the selfassembled structures.

The type of the resulting morphology in the case of SE method is strongly influenced by self-assembly conditions, such as stirring speed and position of a magnetic stir bar. For example, while forming mostly 3D networks under standard solvent evaporation conditions (350 rpm), $\mathrm{E}_{45} \mathrm{C}_{135} \mathrm{M}_{20}$ assembled mostly into spherical and some elongated particles at $500 \mathrm{rpm}$, whereas $100 \mathrm{rpm}$ did not result in self-assembly, but the polymer aggregated into one big piece. Also, when the vial was placed on the side of a stirring plate, and therefore the magnetic stir bar inside located at the side of the vial, 3D networks appeared to be much longer and more branched compared when the vial was at the central position. Some other polymers $\left(\mathrm{E}_{45} \mathrm{C}_{110} \mathrm{M}_{4}, \mathrm{E}_{45} \mathrm{C}_{135} \mathrm{M}_{10}, \mathrm{E}_{45} \mathrm{C}_{135} \mathrm{M}_{25}\right)$ also formed extended parts of networks under such conditions. Thus, the data indicate that the structures formed in solvent evaporation method are under kinetic control.

In co-solvent method, the type of the resulting morphology is sensitive to the preparation procedure. For instance, when THF solution of $\mathrm{E}_{45} \mathrm{C}_{153} \mathrm{M}_{4}$ was added slower $\left(\sim 40 \mu \mathrm{l} \cdot \mathrm{min}^{-1}\right)$ into the aqueous stirring solution, the polymer self-assembled into spherical 
particles, but not into polymersomes as under standard conditions $\left(\sim 200 \mu \mathrm{l} \cdot \mathrm{min}^{-1}\right)$. On the basis of these observations, we concluded that the structures obtained in co-solvent method are under kinetic control.

\section{Transition from kinetically frozen to pseudoequilibrium morphologies.}

Kinetically controlled morphologies may undergo transition towards equilibrium structures, as for example has been demonstrated for poly(acrylic acid)-block-polystyrene (PAA- $b$-PS) system in organic solvent-water mixtures by Eisenberg and co-workers. ${ }^{48-49}$ In the case of PEO- $b$-PCL- $b$-PMOXA polymers the transition from kinetically trapped morphologies obtained in solvent evaporation technique to pseudoequilibrium structures observed in film rehydration method is highly unlikely to happen under ambient temperatures due to the semicrystalline nature of the PCL block: the structures obtained in solvent evaporation method were stable for at least 6 months at room temperature. Therefore, to test such transformation, we incubated at $62{ }^{\circ} \mathrm{C}$ for $24 \mathrm{~h}$ the structures formed by $\mathrm{E}_{45} \mathrm{C}_{110} \mathrm{M}_{4}$, $\mathrm{E}_{45} \mathrm{C}_{135} \mathrm{M}_{4}, \mathrm{E}_{45} \mathrm{C}_{135} \mathrm{M}_{20}$ in solvent evaporation method after their self-assembly. After incubation at $62{ }^{\circ} \mathrm{C}$, the assemblies formed by these polymers partially transferred to the ones formed in film rehydration method (Fig. 8, S7). These findings confirm that (i) the morphologies formed in solvent evaporation method are under kinetic control; (ii) the transition from kinetically trapped to pseudoequilibrium structures is possible above the melting temperature of the PCL block, which allows for its structural rearrangements.
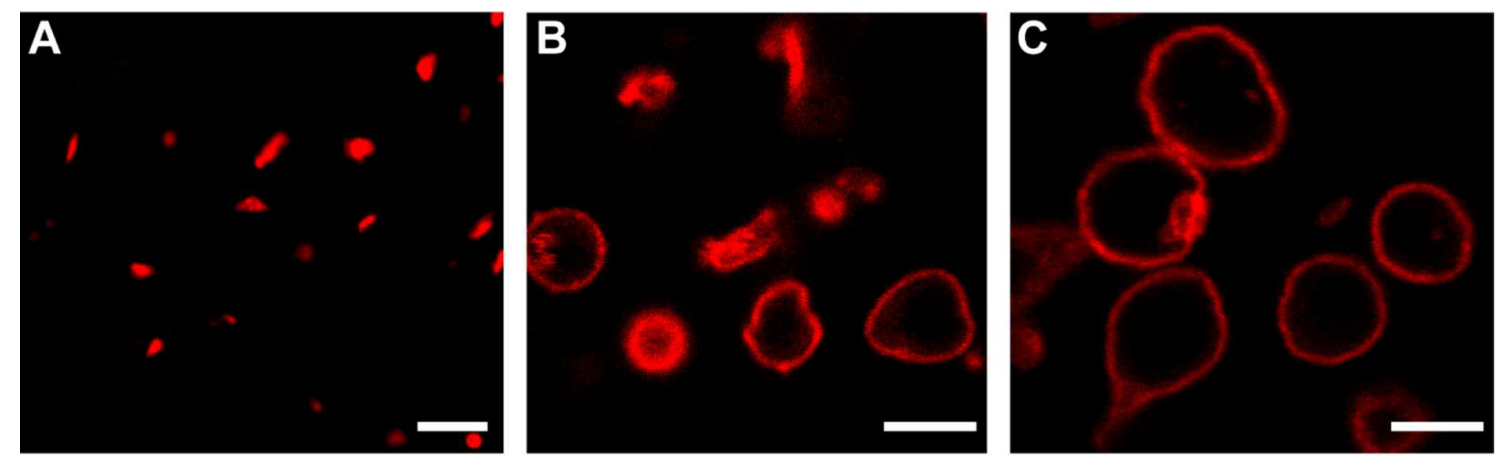
Figure 8. LSM images of the self-assembled structures formed by $\mathrm{E}_{45} \mathrm{C}_{110} \mathrm{M}_{4}$ in different self-assembly methods: (A) solvent evaporation; (B) solvent evaporation followed by incubation at $62{ }^{\circ} \mathrm{C}$ for $24 \mathrm{~h}$; (C) film rehydration. Structures were stained with Bodipy 630/650. Scale bars are $5 \mu \mathrm{m}$.

In solvent evaporation method, elongated structures are the result of the formation of ABC loops at the $\mathrm{CH}_{2} \mathrm{Cl}_{2}-\mathrm{H}_{2} \mathrm{O}$ interface and their further aggregation in aqueous solution upon evaporation of $\mathrm{CH}_{2} \mathrm{Cl}_{2}$ (Scheme 4). Such structures might be not energetically favorable due to a tension at the bending site of the PCL loop and repulsive forces between PEO and PMOXA corona. This could explain why above $\mathrm{T}_{\mathrm{m}}$ of PCL structures obtained in SE method transform into structures observed in film rehydration method, which are energetically more favorable. Solvent evaporation method performed at $62{ }^{\circ} \mathrm{C}$ should also lead to pseudoequilibrium morphologies, but fast evaporation of the organic solvent $\left(\mathrm{CH}_{2} \mathrm{Cl}_{2}\right.$ or $\mathrm{CHCl}_{3}$ ) led to the formation of the polymer layer on top of the aqueous solution preventing it from self-assembly.

In co-solvent method, THF is present during self-assembly and modifies packing geometry of the polymer molecules compared to film rehydration method (Scheme 5). The latter suggests that THF is involved in stabilization of the assembled morphologies. Nevertheless, polymersomes formed by $\mathrm{E}_{45} \mathrm{C}_{153} \mathrm{M}_{11}$ in co-solvent method were stable after a week of the dialysis against water (Fig. 5C, inset). However, after a year of storage at room temperature, these polymersomes collapsed into spherical particles. Such transition was possible presumably due to the presence of residual THF trapped in the hydrophobic core that enabled structural rearrangements of the PCL block. On the contrary to co-solvent method, structures formed in film rehydration method, where organic solvent was removed before self-assembly, were stable for at least a year of storage at room temperature. The true 
equilibrium morphologies for co-solvent method cannot be determined due to the uncertain amount of THF present in the system during storage. 


\section{Conclusions}

We reported new aqueous self-assembled structures of micrometer size for amphiphilic triblock copolymers in the example of PEO-b-PCL- $b$-PMOXA: cloud-like aggregates and 3D networks.

Morphology trends of diblock copolymers PEO- $b$-PCL are maintained globally the same for all used self-assembly methods: polymersomes - spherical particles with increasing $f$. In contrast, morphology trends of triblock copolymers PEO-b-PCL- $b$-PMOXA depend on the self-assembly method and how $f$ is varied - through PCL or PMOXA block.

In film rehydration method system is on the way in achieving global equilibrium, and we refer to the structures formed in this method as pseudoequilibrium morphologies. Solvent evaporation and co-solvent techniques led to the kinetically controlled structures. The kinetically controlled morphologies formed in solvent evaporation method can be transformed, at least partially, to the pseudoequilibrium morphologies above $T_{m}$ of PCL. In co-solvent method, the type of morphology strongly depended on the co-solvent nature.

The presented family of PEO- $b$-PCL- $b$-PMOXA polymers and their self-assembled structures can be relevant for biomedical applications due to biocompatible and proteinrepellent nature of $\mathrm{PEO}^{50}$ and $\mathrm{PMOXA}^{51}$ corona, and biodegradability of PCL core. ${ }^{52-53}$ Elongated structures and 3D networks formed by PEO- $b$-PCL- $b$-PMOXA are uniquely accessible via solvent evaporation technique. These structures are promising candidates for drug delivery purposes, since anisotropic morphologies possess longer blood circulation time compared to the spherical analogues, ${ }^{54}$ and $3 \mathrm{D}$ networks based on biodegradable polymers are of special interest for tissue engineering purposes. ${ }^{55-56}$ In addition, self-assembled structures formed by PEO- $b$-PCL- $b$-PMOXA possess enormous stability due to the semicrystalline nature of the PCL block: the assemblies formed in film rehydration and solvent evaporation methods were stable after at least 6 months of storage at room 
temperature, and the structures stayed intact after shaking and centrifugation at $13000 \mathrm{~g}$ for 1 $2 \mathrm{~h}$.

Polymersomes formed by $\mathrm{ABC}$ triblock copolymers with immiscible A and C blocks should be thermodynamically stable due to different curvature at $\mathrm{A}-\mathrm{B}$ and $\mathrm{C}-\mathrm{B}$ interfaces. To definitely proof this, one needs to investigate additionally formation of polymersomes by other narrowly dispersed $\mathrm{ABC}$ polymers with amorphous B block with low glass transition temperature $\left(<20^{\circ} \mathrm{C}\right)$.

The conclusions drawn from self-assembly of PEO- $b$-PCL- $b$-PMOXA may not necessarily fully apply to other ABC systems in (A, C)-selective solvent. Semicrystalline nature of PCL block is responsible for micrometer sizes of the obtained structures and may dictate the trends in the self-assembly of the tested polymers. Therefore, to establish general rules for ABC self-assembly in (A, C)-selective solvents, similar studies should be performed for other $\mathrm{ABC}$ systems where $\mathrm{B}$ is an amorphous block and the self-assembled structures possess nanometer sizes. In addition, to acquire the full advantage of the complex composition of self-assembled morphologies of PEO- $b$-PCL- $b$-PMOXA, presence of patches and/or Janus corona due to the phase separation of PEO and PMOXA blocks ${ }^{34-35}$ should be investigated. 


\begin{abstract}
Acknowledgments
SNSF, NCCR Molecular Systems Engineering, and the University of Basel are acknowledged for financial support. We thank Carola Alampi (C-CINA, University of Basel) for cryoTEM, Samuel Lörcher (University of Basel) for TEM, and Dr. Alexia LoyntonFerrand (IMCF, University of Basel) for help with z-stack CLSM experiments. E.K. acknowledges Dr. Alina Sekretaryova and Dr. Onur Parlak (Stanford University) for fruitful discussions and useful comments.
\end{abstract}




\section{References}

1. Ball, P., H2O. A Biography of Water. Phoenix. Orion Books Ltd, London: 2000.

2. Discher, D. E.; Ahmed, F., POLYMERSOMES. Annual Review of Biomedical Engineering 2006, 8 (1), 323-341.

3. Penczek, S.; Cypryk, M.; Duda, A.; Kubisa, P.; Słomkowski, S., Living ring-opening polymerizations of heterocyclic monomers. Progress in Polymer Science 2007, 32 (2), 247282.

4. Sedlacek, O.; Monnery, B. D.; Filippov, S. K.; Hoogenboom, R.; Hruby, M., Poly(2Oxazoline)s - Are They More Advantageous for Biomedical Applications Than Other Polymers? Macromolecular Rapid Communications 2012, 33 (19), 1648-1662.

5. Braunecker, W. A.; Matyjaszewski, K., Controlled/living radical polymerization: Features, developments, and perspectives. Progress in Polymer Science 2007, 32 (1), 93-146.

6. Mai, Y.; Eisenberg, A., Self-assembly of block copolymers. Chemical Society Reviews 2012, 41 (18), 5969-5985.

7. Jain, S.; Bates, F. S., Consequences of Nonergodicity in Aqueous Binary PEO-PB Micellar Dispersions. Macromolecules 2004, 37 (4), 1511-1523.

8. Jain, S.; Bates, F. S., On the Origins of Morphological Complexity in Block Copolymer Surfactants. Science 2003, 300 (5618), 460-464.

9. Zhang, L.; Eisenberg, A., Multiple Morphologies of "Crew-Cut" Aggregates of Polystyrene- $<\mathrm{em}>\mathrm{b}</ \mathrm{em}>$-poly(acrylic acid) Block Copolymers. Science 1995, 268 (5218), 1728-1731.

10. Zhang, L.; Eisenberg, A., Formation of crew $\square$ cut aggregates of various morphologies from amphiphilic block copolymers in solution. Polymers for Advanced Technologies 1998, 9 (10 $\square 11), 677-699$.

11. Dionzou, M.; Morere, A.; Roux, C.; Lonetti, B.; Marty, J. D.; Mingotaud, C.; Joseph, P.; Goudouneche, D.; Payre, B.; Leonetti, M.; Mingotaud, A. F., Comparison of methods for the fabrication and the characterization of polymer self-assemblies: what are the important parameters? Soft Matter 2016, 12 (7), 2166-2176.

12. Terreau, O.; Bartels, C.; Eisenberg, A., Effect of Poly(acrylic acid) Block Length Distribution on Polystyrene-b-poly(acrylic acid) Block Copolymer Aggregates in Solution. 2. A Partial Phase Diagram. Langmuir 2004, 20 (3), 637-645.

13. Braun, J.; Bruns, N.; Pfohl, T.; Meier, W., Phase Behavior of Vesicle-Forming Block Copolymers in Aqueous Solutions. Macromolecular Chemistry and Physics 2011, 212 (12), $1245-1254$.

14. Walther, A.; Millard, P.-E.; Goldmann, A. S.; Lovestead, T. M.; Schacher, F.; BarnerKowollik, C.; Müller, A. H. E., Bis-Hydrophilic Block Terpolymers via RAFT Polymerization: Toward Dynamic Micelles with Tunable Corona Properties. Macromolecules 2008, 41 (22), 8608-8619.

15. Zhu, W.; Li, Y.; Liu, L.; Zhang, W.; Chen, Y.; Xi, F., Biamphiphilic triblock copolymer micelles as a multifunctional platform for anticancer drug delivery. Journal of Biomedical Materials Research Part A 2011, 96 A (2), 330-340.

16. Konishcheva, E. V.; Zhumaev, U. E.; Meier, W. P., PEO-b-PCL-b-PMOXA Triblock Copolymers: From Synthesis to Microscale Polymersomes with Asymmetric Membrane. Macromolecules 2017, 50 (4), 1512-1520.

17. Stoenescu, R.; Meier, W., Vesicles with asymmetric membranes from amphiphilic ABC triblock copolymers. Chemical communications (Cambridge, England) 2002, (24), 3016-7. 
18. Liu, F.; Eisenberg, A., Preparation and $\mathrm{pH}$ Triggered Inversion of Vesicles from Poly(acrylic Acid)-block-Polystyrene-block-Poly(4-vinyl Pyridine). Journal of the American Chemical Society 2003, 125 (49), 15059-15064.

19. Mason, A. F.; Thordarson, P., Polymersomes with Asymmetric Membranes Based on Readily Accessible Di- and Triblock Copolymers Synthesized via SET-LRP. ACS Macro Letters 2016, 5 (10), 1172-1175.

20. Wittemann, A.; Azzam, T.; Eisenberg, A., Biocompatible Polymer Vesicles from Biamphiphilic Triblock Copolymers and Their Interaction with Bovine Serum Albumin. Langmuir 2007, 23 (4), 2224-2230.

21. Liu, Q.; Chen, J.; Du, J., Asymmetrical Polymer Vesicles with a "Stealthy" Outer Corona and an Endosomal-Escape-Accelerating Inner Corona for Efficient Intracellular Anticancer Drug Delivery. Biomacromolecules 2014, 15 (8), 3072-3082.

22. Stoenescu, R.; Graff, A.; Meier, W., Asymmetric ABC-triblock copolymer membranes induce a directed insertion of membrane proteins. Macromolecular bioscience 2004, 4 (10), 930-5.

23. Stoenescu, R.; Meier, W., Asymmetric Membranes from Amphiphilic ABC Triblock Copolymers. Molecular Crystals and Liquid Crystals 2004, 417 (1), 185-191.

24. Liu, G.; Ma, S.; Li, S.; Cheng, R.; Meng, F.; Liu, H.; Zhong, Z., The highly efficient delivery of exogenous proteins into cells mediated by biodegradable chimaeric polymersomes. Biomaterials 2010, 31 (29), 7575-7585.

25. Schmalz, H.; Schmelz, J.; Drechsler, M.; Yuan, J.; Walther, A.; Schweimer, K.; Mihut, A. M., Thermo-Reversible Formation of Wormlike Micelles with a MicrophaseSeparated Corona from a Semicrystalline Triblock Terpolymer. Macromolecules 2008, 41 (9), 3235-3242.

26. Walther, A.; Barner-Kowollik, C.; Müller, A. H. E., Mixed, Multicompartment, or Janus Micelles? A Systematic Study of Thermoresponsive Bis-Hydrophilic Block Terpolymers. Langmuir 2010, 26 (14), 12237-12246.

27. Dag, A.; Zhao, J.; Stenzel, M. H., Origami with ABC Triblock Terpolymers Based on Glycopolymers: Creation of Virus-Like Morphologies. ACS Macro Letters 2015, 4 (5), 579583.

28. Barthel, M. J.; Schacher, F. H.; Schubert, U. S., Poly(ethylene oxide) (PEO)-based ABC triblock terpolymers - synthetic complexity vs. application benefits. Polymer Chemistry 2014, 5 (8), 2647-2662.

29. Gröschel, A. H.; Schacher, F. H.; Schmalz, H.; Borisov, O. V.; Zhulina, E. B.; Walther, A.; Müller, A. H. E., Precise hierarchical self-assembly of multicompartment micelles. Nature Communications 2012, 3, 710.

30. Löbling, T. I.; Borisov, O.; Haataja, J. S.; Ikkala, O.; Gröschel, A. H.; Müller, A. H. E., Rational design of $\mathrm{ABC}$ triblock terpolymer solution nanostructures with controlled patch morphology. Nature Communications 2016, 7, 12097.

31. Konishcheva, E.; Häussinger, D.; Lörcher, S.; Meier, W., Key aspects to yield low dispersity of PEO-b-PCL diblock copolymers and their mesoscale self-assembly. European Polymer Journal 2016, 83, 300-310.

32. Qi, W.; Ghoroghchian, P. P.; Li, G.; Hammer, D. A.; Therien, M. J., Aqueous selfassembly of poly(ethylene oxide)-block-poly(?-caprolactone) (PEO-b-PCL) copolymers: disparate diblock copolymer compositions give rise to nano- and meso-scale bilayered vesicles. Nanoscale 2013, 5 (22), 10908-10915.

33. Israelachvili, J. N., Intermolecular and surface forces. Academic press: 2011.

34. Taubert, A.; Furrer, E.; Meier, W., Water-in-water mesophases for templating inorganics. Chemical Communications 2004, (19), 2170-2171. 
35. Casse, O.; Shkilnyy, A.; Linders, J.; Mayer, C.; Häussinger, D.; Völkel, A.; Thünemann, A. F.; Dimova, R.; Cölfen, H.; Meier, W.; Schlaad, H.; Taubert, A., Solution Behavior of Double-Hydrophilic Block Copolymers in Dilute Aqueous Solution. Macromolecules 2012, 45 (11), 4772-4777.

36. Student, The probable error of a mean. Biometrika 1908, 1-25.

37. Smart, T. P.; Mykhaylyk, O. O.; Ryan, A. J.; Battaglia, G., Polymersomes hydrophilic brush scaling relations. Soft Matter 2009, 5 (19), 3607-3610.

38. Rajagopal, K.; Mahmud, A.; Christian, D. A.; Pajerowski, J. D.; Brown, A. E. X.; Loverde, S. M.; Discher, D. E., Curvature-Coupled Hydration of Semicrystalline Polymer Amphiphiles Yields flexible Worm Micelles but Favors Rigid Vesicles: PolycaprolactoneBased Block Copolymers. Macromolecules 2010, 43 (23), 9736-9746.

39. Cameron, N. S.; Corbierre, M. K.; Eisenberg, A., 1998 EWR Steacie Award Lecture Asymmetric amphiphilic block copolymers in solution: a morphological wonderland. Canadian journal of chemistry 1999, 77 (8), 1311-1326.

40. Yu, Y.; Zhang, L.; Eisenberg, A., Morphogenic Effect of Solvent on Crew-Cut Aggregates of Apmphiphilic Diblock Copolymers. Macromolecules 1998, 31 (4), 1144-1154.

41. Bhargava, P.; Zheng, J. X.; Li, P.; Quirk, R. P.; Harris, F. W.; Cheng, S. Z. D., SelfAssembled Polystyrene-block-poly(ethylene oxide) Micelle Morphologies in Solution. Macromolecules 2006, 39 (14), 4880-4888.

42. Bordes, C.; Fréville, V.; Ruffin, E.; Marote, P.; Gauvrit, J. Y.; Briançon, S.; Lantéri, P., Determination of poly( $\varepsilon$-caprolactone) solubility parameters: Application to solvent substitution in a microencapsulation process. International Journal of Pharmaceutics 2010, 383 (1-2), 236-243.

43. Won, Y.-Y.; Brannan, A. K.; Davis, H. T.; Bates, F. S., Cryogenic Transmission Electron Microscopy (Cryo-TEM) of Micelles and Vesicles Formed in Water by Poly(ethylene oxide)-Based Block Copolymers. The Journal of Physical Chemistry B 2002, 106 (13), 3354-3364.

44. Zupancich, J. A.; Bates, F. S.; Hillmyer, M. A., Aqueous Dispersions of Poly(ethylene oxide)-b-poly( $\gamma$-methyl-e-caprolactone) Block Copolymers. Macromolecules 2006, 39 (13), 4286-4288.

45. Adams, D. J.; Butler, M. F.; Weaver, A. C., Effect of Block Length, Polydispersity, and Salt Concentration on PEO-PDEAMA Block Copolymer Structures in Dilute Solution. Langmuir 2006, 22 (10), 4534-4540.

46. Wu, D.; Spulber, M.; Itel, F.; Chami, M.; Pfohl, T.; Palivan, C. G.; Meier, W., Effect of Molecular Parameters on the Architecture and Membrane Properties of 3D Assemblies of Amphiphilic Copolymers. Macromolecules 2014, 47 (15), 5060-5069.

47. He, C.; Sun, J.; Deng, C.; Zhao, T.; Deng, M.; Chen, X.; Jing, X., Study of the Synthesis, Crystallization, and Morphology of Poly(ethylene glycol)-Poly( $\varepsilon$-caprolactone) Diblock Copolymers. Biomacromolecules 2004, 5 (5), 2042-2047.

48. Shen, H.; Eisenberg, A., Morphological Phase Diagram for a Ternary System of Block Copolymer PS310-b-PAA52/Dioxane/H2O. The Journal of Physical Chemistry B 1999, 103 (44), 9473-9487.

49. Zhang, L.; Eisenberg, A., Thermodynamic vs Kinetic Aspects in the Formation and Morphological Transitions of Crew-Cut Aggregates Produced by Self-Assembly of Polystyrene-b-poly(acrylic acid) Block Copolymers in Dilute Solution. Macromolecules 1999, 32 (7), 2239-2249.

50. Knop, K.; Hoogenboom, R.; Fischer, D.; Schubert, U. S., Poly(ethylene glycol) in Drug Delivery: Pros and Cons as Well as Potential Alternatives. Angewandte Chemie International Edition 2010, 49 (36), 6288-6308. 
51. Hoogenboom, R., Poly(2-oxazoline)s: A Polymer Class with Numerous Potential Applications. Angewandte Chemie International Edition 2009, 48 (43), 7978-7994.

52. Goldberg, D., A review of the biodegradability and utility of poly(caprolactone). Journal of environmental polymer degradation 1995, 3 (2), 61-67.

53. Tokiwa, Y.; Calabia, B. P., Biodegradability and Biodegradation of Polyesters. Journal of Polymers and the Environment 2007, 15 (4), 259-267.

54. Geng, Y.; Dalhaimer, P.; Cai, S.; Tsai, R.; Tewari, M.; Minko, T.; Discher, D. E., Shape effects of filaments versus spherical particles in flow and drug delivery. Nat Nano 2007, 2 (4), 249-255.

55. Place, E. S.; George, J. H.; Williams, C. K.; Stevens, M. M., Synthetic polymer scaffolds for tissue engineering. Chemical Society Reviews 2009, 38 (4), 1139-1151.

56. Kweon, H.; Yoo, M. K.; Park, I. K.; Kim, T. H.; Lee, H. C.; Lee, H.-S.; Oh, J.-S.; Akaike, T.; Cho, C.-S., A novel degradable polycaprolactone networks for tissue engineering. Biomaterials 2003, 24 (5), 801-808. 